\title{
Origin and role of water ice clouds in the Martian water cycle as inferred from a general circulation model
}

\author{
F. Montmessin, ${ }^{1}$ F. Forget, ${ }^{2}$ P. Rannou, ${ }^{3}$ M. Cabane, ${ }^{3}$ and R. M. Haberle ${ }^{1}$ \\ Received 22 April 2004; revised 23 June 2004; accepted 18 August 2004; published 9 October 2004.
}

[1] In this paper, we present the results obtained by the general circulation model developed at the Laboratoire de Météorologie Dynamique which has been used to simulate the Martian hydrological cycle. Our model, which employs a simplified cloud scheme, reproduces the observed Martian water cycle with unprecedented agreement. The modeled seasonal evolution of cloudiness, which also compares well with data, is described in terms of the meteorological phenomena that control the Martian cloud distribution. Whereas cloud formation in the tropical region results from seasonal changes in the overturning circulation, Polar Hood clouds are mostly driven by variations of atmospheric wave activity. A sensitivity study allows us to quantify the effects of the transport of water ice clouds on the seasonal evolution of the water cycle. The residence time of cloud particles is long enough to allow cloud advection over great distances (typically thousands of kilometers). Despite the relatively low proportion of clouds $(\sim 10 \%)$ in the total atmospheric inventory of water, their ability to be transported over large distances generally acts at the expense of the north polar cap and generates a water cycle globally wetter by a factor of 2 than a cycle produced by a model neglecting cloud transport. Around aphelion season, clouds modulate the north to south migration of water in a significant fashion and participate just as much as vapor in the cross-equatorial transport of total water. Most of the year, atmospheric waves generate an equatorward motion of water ice clouds near the polar vortex boundaries, partially balancing the opposite poleward flux of water vapor. The combination of both effects delays the return of water to the north polar cap and allows water to build up in the Martian

tropics. INDEX TERMS: 6225 Planetology: Solar System Objects: Mars; 5445 Planetology: Solid Surface Planets: Meteorology (3346); 3367 Meteorology and Atmospheric Dynamics: Theoretical modeling; 0320 Atmospheric Composition and Structure: Cloud physics and chemistry; KEYWORDS: clouds, Mars, water cycle

Citation: Montmessin, F., F. Forget, P. Rannou, M. Cabane, and R. M. Haberle (2004), Origin and role of water ice clouds in the Martian water cycle as inferred from a general circulation model, J. Geophys. Res., 109, E10004, doi:10.1029/2004JE002284.

\section{Introduction}

[2] Water ice clouds in the Martian atmosphere have been identified for decades. However, their restricted occurrence and their low opacity led scientists to consider them minor players in current Mars' climate compared to the global and permanent effect of the dust haze on the thermal structure of the atmosphere. Kahn [1984] documented occurrences and morphologies of condensate clouds from Viking Orbiter images. Recently, similar work has been done with Mars Orbiter Camera (MOC) pictures [Wang and Ingersoll, 2002]. These studies demonstrate the utility of tracking clouds in the Martian

\footnotetext{
${ }^{1}$ NASA Ames Research Center, Moffett Field, California, USA.

${ }^{2}$ Laboratoire de Météorologie Dynamique, Institut Pierre Simon Laplace, Paris, France.

${ }^{3}$ Service d'Aéronomie, Institut Pierre Simon Laplace, Paris, France.

atmosphere, since they can be used to determine wind direction, as well as various meteorological phenomena such as cyclogenesis and large-scale uplifts.

[3] As stated by Richardson et al. [2002], Martian water ice clouds may not fall in the same class of climate players as their terrestrial counterparts. Little, if any, precipitation is expected to occur from these clouds, while their associated latent heat release is theoretically negligible in comparison of radiative heating rates [Savijärvi, 1995]. However, this perception of the climatic impact of Martian water ice clouds is based on a limited data set.

[4] Analysis of Viking Orbiter data has focused mostly on dust aerosols. Only recently have clouds been studied using, for example, the Infrared Thermal Mapping (IRTM) data [Tamppari et al., 2000, 2003]. However, the inclusion of cloud opacity as part of the standard products of the Thermal Emission Spectrometer (TES) instrument [Smith et al., 2001] does attest to a growing concern about Martian clouds in the Mars atmosphere community. Also, Liu et al. [2003] recently made a comparison between IRTM and TES 
meteorological data sets with a specific emphasis on water ice cloud observations.

[5] So far, two major seasonal cloud regimes have been widely recognized:

[6] 1. The Equatorial Cloud Belt (also referred as to the "Aphelion Cloud Belt"), occurs during northern spring and summer and covers the equatorial regions between $10^{\circ} \mathrm{S}$ and $30^{\circ} \mathrm{N}$. This recurrent Martian cloud feature was first identified by Clancy et al. [1996] from ground-based measurements.

[7] 2. The Polar Hoods form in middle to high latitudes of fall/spring hemispheres and appear strongly related to the seasonal evolution of the $\mathrm{CO}_{2}$ caps.

[8] Both events are robust features of the cloud annual cycle [Liu et al., 2003], with very little interannual variability recorded for the Equatorial Cloud Belt.

[9] Scientific interest in the role of clouds on the Martian climate system has been expressed in a number of publications. Kahn [1990] advocated clouds as part of a process leading to an enhanced sequestration of water in the regolith. This mechanism was suggested to explain the premature moistening of the midlatitude regions at a season where the north permanent cap sublimation had not yet begun. Following their first observation of what has been since called the "Aphelion cloud belt," Clancy et al. [1996] proposed a mechanism, involving clouds, to predict preferred storage location of water with changes of perihelion date. The so-called Clancy effect comes from the potential ability of clouds to confine water below the return branch of the solsticial Hadley cell if the latter is synchronized with the aphelion season. In practice, aphelion season implies lower atmospheric temperatures in the tropics and thus lower levels of condensation/precipitation. As particle sedimentation tends to confine water at low levels, water would be forced to remain essentially in the aphelion summer tropics, preventing it from being carried into the Southern Hemisphere. Perihelion season, on the contrary, comes with enhanced solar forcing, a dustier atmosphere and warmer temperatures, which allow water to be carried by the Hadley cell toward the winter hemisphere without experiencing the effects of cloud sedimentation. On an annual average, Clancy et al. [1996] suggest a net flux of water toward the hemisphere for which summer occurs near aphelion. The current orbital configuration should therefore favor the Northern Hemisphere. It also implies that this situation is reversed when the perihelion is shifted of $180^{\circ}$. Clancy et al. [1996] even suggested that the current position of the permanent water ice cap is a consequence of this effect.

[10] There have been several papers focusing specifically on the role of clouds in the water cycle using climate models. James [1990] included a condensed phase in his original 1-D (latitude) diffusion model (published first by James [1985]) to investigate its effect on the water cycle. He found that the Polar Hoods were important components of the water cycle though the cloud amounts simulated were much higher than expected. Perennial midlatitude ground ice was also an unrealistic prediction of his model. Though highly parameterized, James' model highlighted the importance of transport and precipitation in determining the net annual transfer of water from north to south.

[11] The first work to include "true" microphysical processes like sedimentation, came with Richardson and
Wilson [2002] and their description of the water cycle equilibration mechanism based on a three-dimensional general circulation model. These authors wrote a later paper specifically dedicated to water ice clouds and their contribution in the main processes of the water cycle Richardson et al. [2002]. However, except in the case of the prescription of an unrealistically large cloud particle radius, their model was unable to match Viking observations, especially the actual ratio of humidity between the northern and the southern hemisphere.

[12] Böttger [2003] interpreted the need for excessively large particles in Richard and Wilson's work as an indication of the need for an adsorbing regolith. He expanded on Richardson and Wilson's work by conducting GCM simulations with the Oxford model using an adsorbing regolith based on the model of Zent et al. [1993]. He found that it was possible to reproduce the observed water cycle using much smaller cloud particles only if an adsorbing regolith was included in the model. Böttger's cloud scheme, however, did not predict cloud particle size. As in Richardson and Wilson's model, Böttger's cloud particle sizes were prescribed and monodisperse. As we will show, very different results can be obtained when cloud particle size is predicted.

[13] The first assessment of cloud radiative effects was made by Haberle et al. [1999] and Colaprete and Toon [2000], who both showed that cloud infrared emission could be responsible for the observed inversion in the Pathfinder descent temperature profile. In addition, Rodin et al. [1999] emphasized the strong interactions between dust and clouds which could significantly affect the Martian climate. In particular, these authors show how these interactions could put the climatic system into a precarious state of equilibrium during the cloudy aphelion season. Finally, Hinson and Wilson [2004] have recently demonstrated that cloud radiative feedback can couple with dynamical phenomena to amplify the signature of thermal tides on the temperature structure. Such a study provides another example of the importance of water ice clouds in the current Martian climate system.

[14] In this paper, we build on these earlier works and give a more comprehensive description of the role of clouds in the Martian hydrological cycle by assessing their ability to act as a mobile reservoir for water. We base our discussion on the results of a 3-D Martian general circulation model (GCM) for which clouds receive a realistic, though simplified, representation. The main issues we address are: What are the mechanisms that control the observed cloud distribution? Where and when is cloud transport significant? What is the magnitude of their effect on cross-equatorial transport of total water? In answering these questions we show that clouds are major players in determining the current seasonal and spatial distribution of water in the atmosphere and at the surface. In the next section, we describe the model used for this study. We then compare model results with available observations. We show that the current Mars water cycle, as observed by TES, can be reproduced despite the absence of a regolith scheme and with reasonable assumptions for cloud sedimentation. Finally, we give a complete assessment of the role of clouds in the water cycle by conducting sensitivity studies involving various parameterizations of cloud- 
related processes. Here, we not only quantify the effect of the Aphelion Cloud Belt on the water vapor asymmetry between hemispheres, but we also discuss the impact of the Polar Hoods on the latitudinal distribution of humidity.

\section{Model Description}

\subsection{Climate Model}

[15] We use the Mars General Circulation Model (MGCM) developed at Laboratoire de Météorologie Dynamique (LMD). A detailed description is given by Forget et al. [1999]. This MGCM is a three-dimensional grid point dynamic model based on the terrestrial LMD model [Sadourny and Laval, 1984].

[16] The model solves both the primitive equations of meteorology and the radiative transfer equation in visible and thermal infrared bands. Martian topography is based on MOLA data [Smith et al., 1999], whereas surface properties of the Martian soil (thermal inertia and albedo) were taken from IRTM and TES observations. The thermal inertia map used by the GCM is a composite of several data sets. TES inversions of Mellon et al. [2000] have been employed to fill the region between $30^{\circ} \mathrm{S}$ and $60^{\circ} \mathrm{N}$, whereas the values of Palluconi and Kieffer [1981] were taken for the region between $30 \mathrm{~S}$ and $60 \mathrm{~S}$. Constraints on albedo are inferred from the work of Pleskot and Miner [1981] except in the Polar Regions where the results of Paige et al. [1994] and Paige and Keegan [1994] for both albedo and thermal inertia are employed. The thermophysical properties of the polar regions have been recently reprocessed by Vasavada et al. [2000] in order to account for an atmospheric component (mostly dust) in the retrieval. Vasavada et al. [2000] note that the effect of including the dust contamination in the retrieval leads to a significant decrease of the derived thermal inertia values. For similar reasons, the thermal inertia data set used by the model has been obtained by reducing the Paige et al.'s data of approximately $25 \%$ in both polar regions [Forget et al., 2001]. We note, however, that our model is able to match the observed seasonal evolution of the north polar surface temperatures given by Bass and Paige [2000].

[17] The coordinate system is terrain-following, where the $\sigma$ coordinate is used to characterize level altitudes (where $\sigma$ is pressure divided by surface pressure). The typical model configuration has 25 vertical levels, yielding a model top above $80 \mathrm{~km}$. However, the first three layers are respectively set at about $4 \mathrm{~m}, 19 \mathrm{~m}$ and $44 \mathrm{~m}$, in order to obtain a fine representation of the boundary layer. All the results presented throughout this paper have been obtained with a spatial configuration of " $64 \times 48$," i.e., 64 points on every latitudinal circle and 48 points on every meridian. This gives a resolution of 5.6 degree of longitude and 3.8 degree of latitude.

[18] Radiative transfer representation includes absorption by the strong $\mathrm{CO}_{2} 15 \mu \mathrm{m}$ absorption band and parameterizes $\mathrm{CO}_{2}$ absorption in the near-infrared, an effect which is theoretically significant at altitudes greater than $50 \mathrm{~km}$. Radiative transfer also accounts for absorption and scattering of solar radiation by dust particles, and their absorption/ emission and multiple scattering of thermal radiation. Considering the relatively dry Martian atmosphere, water vapor is ignored in the radiative budget [Savijärvi, 1991].
[19] In our simulations, dust particles are not allowed to be moved by the GCM resolved winds. Instead, dust mass mixing ratio is forced to follow a spatial and temporal distribution that has been developed by Forget et al. [2001]. Basically, dust mixing ratio is set constant from the surface up to a given elevation $\left(z_{\max }\right)$ above which it rapidly declines. This is a common approach in many GCMs where dust is supposed to follow a vertical profile in equilibrium with both sedimentation and mixing process [Conrath, 1975]. According to this approximation, the dust mass mixing ratio at a pressure level $p$ is given by

$$
q=q_{o} \exp \left\{\nu\left[1-\left(\frac{p_{\text {ref }}}{p_{s}}\right)^{70 / z_{\max }}\right]\right\},
$$

where $q_{o}$ is a constant determined by the prescribed opacity at a reference pressure level $p_{\text {ref }}(\sim 7 \mathrm{mbar}), p_{s}$ is the surface pressure, $v$ is the so-called Conrath parameter and $z_{\max }$ is given in $\mathrm{km}$. For pressure levels higher than $p_{\text {ref }} q$ is set equal to $q_{o}$. Note that in our simulations, the Conrath parameter $v$ remains always constant at a value of 0.007 .

[20] As dust constitutes the only adjustable radiative constituent in the model, Forget et al. [2001] designed the "MGS dust scenario" in order to fit most of the thermal profiles observed during the Mars Global Surveyor (MGS) mission. To do so, Forget et al. [2001] prescribed a latitudinally and seasonally varying dust content, as well as a latitudinally and seasonally varying $z_{\max }$. The latter obeys the following expression:

$$
\begin{aligned}
z_{\max }\left(\theta, L_{s}\right)= & 60+18\left(\sin \left(L_{s}-158\right)\right) \\
& -\left(32+18 \sin \left(L_{s}-158\right)\right) \sin ^{4} \theta \\
& -8 \sin \left(L_{s}-158\right) \sin ^{5} \theta
\end{aligned}
$$

where $\theta$ is the latitudinal coordinate in degrees and $L_{s}$ is the solar longitude in degrees. The column integrated dust opacities in the northern and the southern hemispheres (respectively $\tau_{\text {north }}$ and $\tau_{\text {south }}$ ) vary seasonally and spatially according to

$$
\begin{aligned}
& \tau_{\text {north }}\left(\theta, L_{S}\right)=\tau_{n}+0.5\left(\tau_{\text {eq }}-\tau_{n}\right)(1-\tanh (4.5-\theta / 10)), \\
& \tau_{\text {south }}\left(\theta, L_{s}\right)=\tau_{s}+0.5\left(\tau_{e q}-\tau_{s}\right)(1+\tanh (4.5-\theta / 10)) .
\end{aligned}
$$

$\tau_{\text {north }}$ and $\tau_{\text {south }}$ are given at a reference pressure of $7 \mathrm{mbar}$, whereas $\tau_{n}, \tau_{s}$ and $\tau_{e q}$ are yielded by the following expressions:

$$
\begin{gathered}
\tau_{n}=0.1, \\
\tau_{e q}=0.2+0.3\left(\cos \left(0.5\left(L_{s}-250\right)\right)\right)^{14}, \\
\tau_{s}=0.1+0.4\left(\cos \left(0.5\left(L_{s}-250\right)\right)\right)^{14} .
\end{gathered}
$$

The resulting behavior of both dust opacity and $z_{\max }$ as "seen" by our MGCM is shown in Figure 1 .

[21] Since it has been designed in this scope, the "MGS dust scenario" provides a very consistent reproduction of the Martian meteorological environment. This 

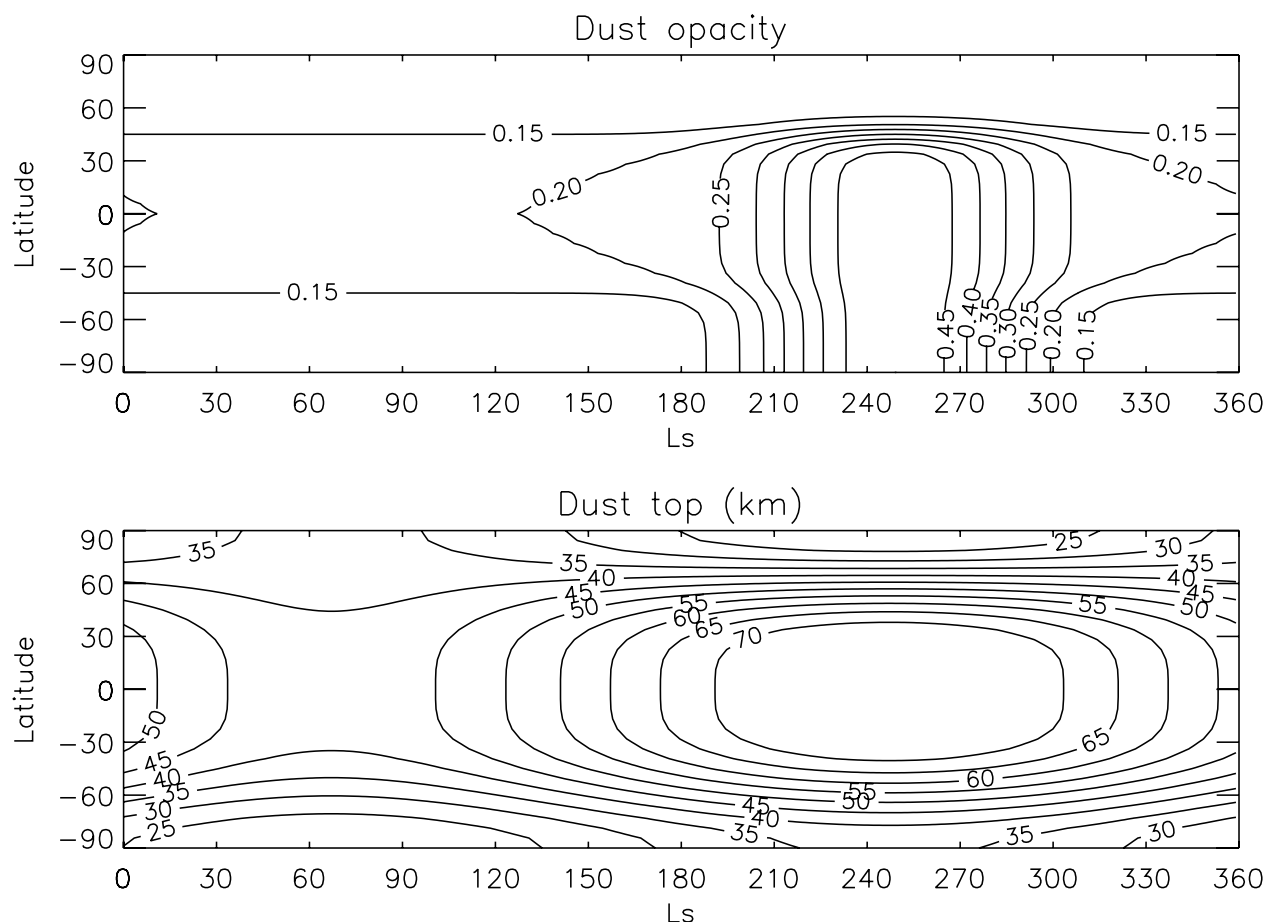

Figure 1. (top) Seasonal and latitudinal distribution of dust opacity used by the model. (bottom) Associated height of the dust "top" in kilometers, where the dust mass mixing ratio drops to $1 / 1000$ th of its value at 7 mbar.

tuning of the model offers a significant advantage when modeling species like clouds which have a nonlinear dependence on temperature. On the other hand, fixing the dust spatial distribution means we are ignoring dustwater ice cloud interactions.

\subsection{Tracer-Related Processes and Simulation Settings}

[22] Our cloud study takes place within the more general context of the Martian water cycle. As such, the model has representations of the major processes affecting water vapor and clouds.

\subsubsection{Sublimation Scheme}

[23] Water exchanges with the surface are assumed to obey the classical equation already used on Mars by Flasar and Goody [1976]:

$$
E_{w}=\rho C_{d} u *\left(q_{v g}-q_{v a}\right),
$$

where $E_{w}$ is the turbulent flux of water at the base of the atmosphere, $\rho$ is the atmospheric density near the ground, $C_{d}$ is the drag coefficient, $u_{*}$ is the friction velocity $\left(=\sqrt{u^{2}+v^{2}}\right.$, with $u$ and $v$ the zonal and meridional wind intensities in the first atmospheric layer), $q_{v g}$ is assumed to be the saturation mass mixing ratio at the ground temperature, and $q_{v a}$ is the actual mass mixing ratio of water vapor at the mid point of the bottom layer (located approximately 4 meters above the surface). Assuming that the wind profile in the lowest part of the atmosphere is logarithmic, $C_{d}$ is taken as [Forget et al., 1999]

$$
C_{d}=\left(\frac{\kappa}{\ln \frac{z}{z_{o}}}\right)^{2}
$$

where $\kappa$ is the Von Karman constant $(\kappa=0.4)$ and $z_{o}$ is the roughness coefficient $\left(z_{o}=0.01 \mathrm{~m}\right)$. This equation implies that the moisture flux subliming off the ground depends not only on the gradient of specific humidity between the surface and the atmosphere, but also on the strength of turbulent mixing near the ground. It is worth noting that Richardson and Wilson [2002] used a formulation for $E_{w}$ that was previously employed by Haberle and Jakosky [1990]. The latter accounts for an additional buoyancy term generated by the presence of lighter water molecules below those of $\mathrm{CO}_{2}$.

\subsubsection{Microphysical Processes}

[24] Representing microphysics in a Martian GCM is mostly an issue of timescale and number of tracers. Michelangeli et al. [1993] have shown that nucleation and condensation processes may occur on timescales of the order of seconds or less. Using our GCM time step of 30 minutes would therefore not permit an accurate calculation of these processes.

[25] Most of the microphysical models published so far [Michelangeli et al., 1993; Colaprete et al., 1999; Montmessin et al., 2002] for the Martian atmosphere solve the continuity equation of aerosols using a significant number of size bins (usually several tens) to properly reproduce the aerosol particle size distribution. If this kind of microphysics scheme was introduced into a GCM, the required number of independent tracers would be proportional to that of size bins, dramatically increasing computation time. Coupling this type of scheme to a GCM becomes excessively time consuming when multiannual simulations or sensitivity studies are conducted. Nonetheless, Rodin [2002] has recently proposed an elegant way to overcome this issue. Rodin [2002] shows that a particle distribution can be advantageously represented by its first 
three moments, therefore limiting the number of tracers used to describe aerosol populations in Martian GCMs. This approach should provide an efficient alternative in our future GCM studies which will be more specifically focused on microphysical interactions between dust and clouds.

[26] Recently, Richardson and Wilson [2002] have presented water cycle simulation results using a simplified method to handle cloud formation. Richardson and Wilson [2002] made the assumption that any supersaturated excess of water vapor is instantaneously turned into atmospheric water ice. Moreover, these authors make the additional simplification of a monodisperse population for water ice particles and set the cloud particle radius to a constant value suggested by observations. This type of cloud scheme allows for fast computations, but without comparison with a more sophisticated model it is difficult to determine how accurate this assumption is. One may argue, for example, that using a single value for particle size cannot reproduce the potential variety of Martian cloud microphysical properties. Although current estimates of cloud particle size are very sparse and restricted to a few regions and seasons, they already exhibit significant variations between northern summer and other seasons [Clancy et al., 2003].

[27] Our approach is similar to that of Richardson and Wilson [2002] in that dust-water ice interactions are not represented. Our model carries only two tracers: water vapor and atmospheric water ice. However, Rossow [1978], in his microphysical study of the Martian atmosphere, stated that the microphysical properties of clouds are mainly controlled by the number of available nuclei to condense onto. With this in mind, we have designed our model to capture the importance of dust nuclei in determining cloud particle size. In particular, we have designed a cloud scheme that predicts cloud particle size.

[28] To first order, we assume that the cloud particle size distribution is monodisperse. The mass mean radius $r_{c}$ can then be deduced from the total mass $M_{c}$ of water ice at a given atmospheric level, the number of nuclei $\mathcal{N}$, and the mean radius of dust nuclei $r_{o}$ :

$$
r_{c}=\left(\frac{M_{c}}{(4 / 3) \pi \rho_{i} \mathcal{N}}+r_{o}^{3}\right)^{1 / 3}
$$

where $\rho_{i}$ is the density of water ice $\left(917 \mathrm{~kg} \cdot \mathrm{m}^{-3}\right)$. The variation of $M_{c}$ depends not only on the relative humidity predicted by the model, but also on the value of $r_{c}$. Indeed, as described by Montmessin et al. [2002],

$$
\frac{d r_{c}}{d t}=\frac{S-S_{e q}}{r_{c}\left(R_{c}+R_{d}\right)}
$$

where $\mathrm{S}$ is the current saturation ratio of water vapor, $S_{e q}$ is its equilibrium value accounting for curvature effect, and $R_{c}$ and $R_{d}$ constitute the heat and diffusive resistances opposed to radius variation. Formal expressions of $R_{c}$ and $R_{d}$ are given by Montmessin et al. [2002]. From equation (3), we can deduce the variation of $M_{c}$ as

$$
d M_{c}=4 \pi \mathcal{N} r_{c}^{2} d r_{c}=4 \pi \mathcal{N} r_{c}^{2} \frac{S-S_{e q}}{r_{c}\left(R_{c}+R_{d}\right)} d t
$$

This determination of $d r_{c}$ more adequately reflects some of the severe constraints imposed by conditions of low pressure and/or low temperature (both increasing substantially the value of $R_{d}$, thus decreasing $d r_{c} / d t$ ) on condensation process. As mentioned above, condensation timescales may, on some occasions, be much shorter than a GCM time step $(\Delta t)$. One of the potential consequences of integrating cloud particle growth rates over a long time step is to generate unphysical transitions from a supersaturated to a subsaturated state and vice-versa. To correct for this effect, we employ the implicit formulation described by Jacobson [1997] to solve the growth rate equation. Indeed, $d M_{c}$ is computed using the $t+1$ value of $S$. Mass conservation imposes that

$$
M_{v, t+1}+M_{c, t+1}=M_{v, t}+M_{c, t},
$$

where subscripts $v$ and $c$ refers to vapor and cloud respectively, and subscripts $t$ and $t+1$ refers to the values of $M$ at the beginning and the end of integration. Since $S=$ $M_{v, t+1} / M_{v, S}$ where $M_{v, S}$ is the mass mixing ratio of water vapor at saturation, we have

$$
M_{v, t+1}=\frac{M_{v, t}+\Delta t 4 \pi \mathcal{N} \rho_{i} r_{c} S_{e q}\left(R_{c}+R_{d}\right)^{-1}}{1+\Delta t 4 \pi \mathcal{N} \rho_{i} r_{c}\left(R_{c}+R_{d}\right)^{-1} M_{v, S}^{-1}} .
$$

Further, we add the additional constraint that $M_{v, t+1}$ can not exceed $M_{v, t}+M_{c, t}$. Once $M_{v, t+1}$ is determined, its value is introduced into the expression yielding $d M_{c}$ and the mass of exchanged water can subsequently be obtained.

[29] Since dust is not transported, we have prescribed $\mathcal{N}$ and $r_{o}$ as follows:

$$
\mathcal{N}(z)=\frac{\tau}{\tau_{\text {ref }}} \mathcal{N}_{o} \exp (-z / H)
$$

where $\tau$ is the total dust optical depth in the column (values of $\tau$ are prescribed by the "MGS dust scenario"), $\tau_{\text {ref }}$ is the reference optical depth $(\sim 0.1)$ which corresponds $\mathcal{N}_{o}$, the reference dust number density near the surface $\left(\sim 2.10^{6} \mathrm{~m}^{-3}\right)$, and $H$ is the atmospheric scale height ( $\sim 10 \mathrm{~km}$, dust is assumed to be uniformly mixed in height). Finally, $r_{o}$ is given by

$$
r_{o}(z)=r_{z o} \exp (-z / h),
$$

where $r_{z o}$ is $0.8 \mu \mathrm{m}$ and $\mathrm{h}$ is $18 \mathrm{~km}$. This simple formulation for $r_{o}$ approximates the effect of sedimentation on the dust particle size profile since large particles fall faster than small ones. At $20 \mathrm{~km}, r_{o}$ is roughly equal to $0.3 \mu \mathrm{m}$. Values of $h$ and $r_{z o}$ have been calibrated using our 1-D reference microphysical scheme presented by Montmessin et al. [2002].

[30] As for sedimentation process, we employ the usual Stokes-Cunningham relationship giving the particle fall velocity $\omega$ already used by Montmessin et al. [2002]:

$$
\omega=\frac{2}{9} \frac{r_{c}^{2} \rho_{i} g}{\eta_{a}}(1+\alpha K n)
$$


where $g$ is Martian gravity (3.72), $\eta_{a}$ is the dynamic viscosity of the air, $K n$ is the Knudsen number, and $\alpha$ is a correction factor given by

$$
\alpha=1.246+0.42 \exp \left(\frac{-0.87}{K n}\right)
$$

In the expression of $\omega$, we use a value of $r_{c}$ corrected by a factor $f$ which accounts for the dispersion of the particle size distribution. A similar approach was employed by Schulz et al. [1998] in the case of dust desert aerosols. Indeed, if we assume that the cloud particles have a log-normal size distribution, we can derive an effective radius that can be used to compute the sedimentation flux of the entire size distribution. This flux can be written as

$$
\mathcal{F}_{\text {sed }}=\rho_{a} \int_{0}^{\infty} n(r) \omega(r) \rho_{i} \frac{4}{3} \pi r^{3} d r
$$

where $\rho_{a}$ is the air density and $n(r)$ the population density. Using a more convenient formulation for $\omega\left(A r^{2}+B r\right.$ where $A$ and $B$ are constant), the latter expression can be rewritten as

$$
\mathcal{F}_{\text {sed }}=\rho_{a} \rho_{i} \frac{4}{3} \pi\left[A \int_{0}^{\infty} n(r) r^{5} d r+B \int_{0}^{\infty} n(r) r^{4} d r\right] .
$$

One of the convenient properties of the log-normal distribution is that

$$
\int_{0}^{\infty} r^{k} n(r) d r=\mathcal{N} r_{o}^{k} \exp \left(0.5 k^{2} \sigma_{o}^{2}\right)
$$

where $\sigma_{o}$ is the standard deviation of the distribution, and $r_{o}$ is the number median radius $\left(=r_{c} \exp \left(-1.5 \sigma_{o}^{2}\right)\right.$. We can now rewrite $\mathcal{F}_{\text {sed }}$ as

$$
\begin{aligned}
\mathcal{F}_{\text {sed }}= & \rho_{a} \rho_{i} \mathcal{N}_{\frac{1}{3}}^{4} \pi r_{o}^{3} \exp \left(4.5 \sigma_{o}^{2}\right)\left[A r_{o}^{2} \exp \left(9 \sigma_{o}^{2}\right)+B r_{o} \exp \left(4.5 \sigma_{o}^{2}\right)\right] \\
& \cdot \exp \left(-\sigma_{o}^{2}\right)
\end{aligned}
$$

to finally obtain

$$
\mathcal{F}_{\text {sed }}=\rho_{a} M_{c}\left[\omega\left(f r_{c}\right) \exp \left(-\sigma_{o}^{2}\right)\right]
$$

where $f$ is equal to $\exp \left(3 \sigma_{o}^{2}\right)$. This enhancement factor $f$ simply reflects the fact that, for a given distribution, the effective sedimentation radius is biased toward larger particles, since the latter fall faster, carry more mass and therefore account for the bulk of the aerosol mass transport.

[31] $f$ has been used as a free parameter in order to obtain the most realistic water cycle with respect to TES observations. In particular, we have focused on the model ability to reproduce the observed seasonal evolution of the total amount of water in each hemisphere [Smith, 2002]. We made $f$ vary in the range $[1.3,1.7]$ corresponding to an effective variance bounded by 0.1 and 0.2 , which are likely values for the water ice particle distribution. During this calibration phase, the model showed a great sensitivity to changing $f$, with the highest value of $f(1.7)$ providing a water cycle about $20 \%$ too dry and the lowest value yielding a water cycle $30 \%$ too wet. This is probably excessive since in reality some processes not represented here (e.g., regolith exchange, cloud radiative feedback) would buffer this sensitivity. However, the value of $f$ providing the most satisfactory fit to data turned out to be 1.5 , corresponding to an effective variance of 0.15 (giving $\sigma_{o} \sim 0.37$ ).

\subsubsection{Tracer Transport}

[32] The MGCM dynamical core takes advantage of a built-in advection scheme (based on a Van-Leer formulation [Hourdin and Armengaud, 1999]), which is used to solve the transport of tracers by the model resolved winds. In addition, water vapor and water ice clouds are vertically redistributed in a way depending on the turbulent kinetic energy diagnosed by the model in each grid box. This vertical transport, akin to a diffusion process, is a typical representation of "eddy mixing." In addition, the model also uses a standard energy conserving convective adjustment scheme which rapidly mixes heat, momentum and tracers in convectively unstable layers.

[33] In its 1-D version, our simplified cloud scheme has been tested against our reference microphysical scheme. The purpose of this experiment was to assess how a simple model assuming a monodisperse population for cloud particle might compare with a model with 50 size bins to describe the evolution of aerosol size distribution. To allow strict comparison between processes common to both schemes (condensation and sedimentation), we forced the nucleation process in our reference model to be activated at negligible supersaturation, allowing dust particles of any size to serve as a condensation nuclei. We found that, in general, our simple cloud scheme was able to reproduce the vertical profiles of water ice and vapor predicted by our reference microphysical scheme, with differences between them less than $50 \%$. The absence of knowledge on the Martian dust nucleation efficiency, a parameter that controls in large part the simulated cloud amount [Michelangeli et al., 1993], implies that even the most sophisticated cloud modeling still suffers from large uncertainties. Hence we feel that our simple cloud scheme provides a satisfactory level of performance given its very low computational cost.

\subsubsection{Simulation Settings}

[34] The presence of the permanent water ice cap at the north pole is represented by an initial amount of ground ice northward of $80^{\circ} \mathrm{N}$. In order to simulate the presence of the residual $\mathrm{CO}_{2}$ cap at the south pole, we constrain the surface temperature of the model southward of $85^{\circ} \mathrm{S}$ to be equal to the phase change temperature of $\mathrm{CO}_{2}$. As stated by Richardson and Wilson [2002] and Houben et al. [1997], this approach is expected to roughly represent the coldtrapping effect the south residual cap has on the overlying water vapor.

[35] Albedo feedback induced by water ice frost on the ground is undoubtedly a difficult issue to address within the context of our simulations. Bare soil albedo might be changed by ice deposition in a way depending on frost grain size and dust content. Until more work is done, our treatment follows that of Richardson and Wilson [2002] in which the surface albedo is set to 0.4 when a water ice layer thicker than $5 \mu \mathrm{m}$ is diagnosed by the model. Exceptions occur in both polar cap regions where the albedo is unchanged, and also in regions covered by $\mathrm{CO}_{2}$ ice. In that case, the $\mathrm{CO}_{2}$ ice albedo prevails. We feel, however, that 
this crude representation of albedo feedback should be substantially improved in the future, especially when considering the importance albedo has on surface temperature and thus on water sublimation and deposition [Bass et al., 2000].

[36] Exchange between the atmosphere and the subsurface have been neglected. Results shown by Richardson and Wilson [2002] for their active regolith simulations suggest that the regolith helps water to build away from the north polar region. In the study made by Böttger [2003], regolith adsorption appears critical to successfully reproduce the seasonal evolution of the water cycle. Unfortunately, there are large uncertainties in the regolith adsorption capacity, which undoubtedly vary regionally with changing soil properties. This makes it difficult to assess what role the regolith actually plays in the water cycle. Our approach here is to ignore the regolith for now and focus first on the role of clouds. As we will show, it is possible to obtain realistic water cycles without including an adsorbing regolith. This does not mean the regolith has no role in the water cycle since our cloud scheme, though an improvement over previous schemes, is still rather crude (e.g., condensation nuclei are prescribed). However, it does emphasize the importance of improving the fidelity of atmospheric processes in the models since these ultimately determine the vertical distribution of water vapor, which in turn will determine any potential exchange with a regolith.

[37] As estimated by Jakosky [1983], a spin-up timescale of several years is necessary for dynamical processes to set up a latitudinal gradient in response to polar water abundances, themselves controlled by local thermodynamical conditions. However, all the simulations presented hereafter are initialized with a moist atmosphere. Mass mixing ratios of water vapor are set to decrease linearly with latitude, where $150 \mathrm{ppm}$ ( $30 \mathrm{pr} . \mu \mathrm{m}$ in column integrated abundance) are imposed at the north pole and 0 at the south pole. This initial background of humidity in the atmosphere allows the water cycle to achieve a steady-state much faster than a simulation where the atmosphere is initially dry, the gain being of several years. We have assumed that a global inventory of water vapor changing interannually only by one percent is at a pseudo-equilibrium state ("pseudo" referring to the fact that a seasonal flux of moisture is permanently trapped by the south polar cap). Indeed, if the water vapor content were to increase geometrically at $1 \%$ per year, the atmosphere would only be $10 \%$ wetter after 10 years. Once this value of $1 \%$ is reached, we stop the simulation and analyze the last year.

\section{Model Results}

[38] The purpose of this section is twofold. First, we would like to demonstrate the ability of the MGCM to reproduce the seasonal behavior of the Martian water ice clouds as inferred from spacecraft observations (in particular, the MGS TES data). We will describe cloud occurrences predicted by the model and we will attempt to provide an explanation of their behavior. In the other part of this section, we will introduce the results obtained with another version of the model where cloud representation has been deliberately simplified. Comparison between these two versions will support the analysis carried out in the next section where the role of clouds in the Martian cycle is discussed in more detail.

[39] Since water ice clouds are a strong function of both temperature and water supply, a successful reproduction of the Martian cloud reservoir and its seasonal evolution depends on how well both the thermal and humidity environments are simulated by the model. As explained previously, use of the "MGS dust scenario" should help ensure that the simulated thermal structure is reasonably well reproduced. Regarding the issue of humidity and by extension the water cycle as a whole, Richardson et al. [2002] show the latter to be sensitive to cloud sedimentation rates and therefore to cloud properties. This intimate coupling between water vapor and cloud ice makes it challenging to predict both values "right" simultaneously.

\subsection{Annual Cycle of Water: Description and Model Results}

[40] Figure 2 shows the TES observations of column water vapor from [Smith, 2004] along with the results of the model from year 6 of the simulation. In what follows we draw heavily from the analysis of [Houben et al., 1997; Richardson and Wilson, 2002] in explaining the seasonal and geographical distribution of water vapor.

[41] Overall, our model provides a good match of the observed water cycle. A prominent peak in water vapor occurs over the north polar region that starts at the end of spring and lasts until midsummer. This peak is associated with intense sublimation of the north polar cap while it is exposed to summer solar radiation. Abundances as high as 90 pr. $\mu \mathrm{m}$ are seen by TES [Smith, 2002] and MAWD [Jakosky and Farmer, 1982]; values larger than 50 pr. $\mu \mathrm{m}$ were are also seen by Sprague et al. [2001] using groundbased measurements. Considering the likely absence of other water sources of this scale, extraction of water vapor from the north pole at this season is of critical importance for the global moistening of the atmosphere.

[42] Haberle and Jakosky [1990] investigated the mechanisms allowing water vapor that sublimes off the cap to be incorporated into the global circulation. Except for unlikely conditions, their 2-D circulation model failed at reproducing the Mars Atmospheric Water vapor Detector (MAWD) observations. The simulated equatorward transport of water appeared insufficient to compare with data. In contrast, 3-D circulation models were more successful at reproducing it, suggesting a major role of the nonzonal circulation in extracting moisture from the north pole. As suggested by Richardson and Wilson [2002], despite its weakness, the 3 -D horizontal mixing in the presence of a latitudinal gradient of humidity allows most of the water to reach northern tropics where it becomes incorporated into the solsticial Hadley cell.

[43] More interesting is the description of the mechanism by which water returns to the permanent cap. Richardson and Wilson [2002] found that, shortly before the sublimation season, the north polar cap has regained most of the water lost during spring and summer. As indicated in Figure 2, water vapor abundances in the northern tropics tend to decrease from the end of summer until next spring. Meanwhile, surface water ice (not shown) accumulates at middle to high latitudes as the season progresses, even during the seasonal cap recession. Quite possibly, baroclinic 

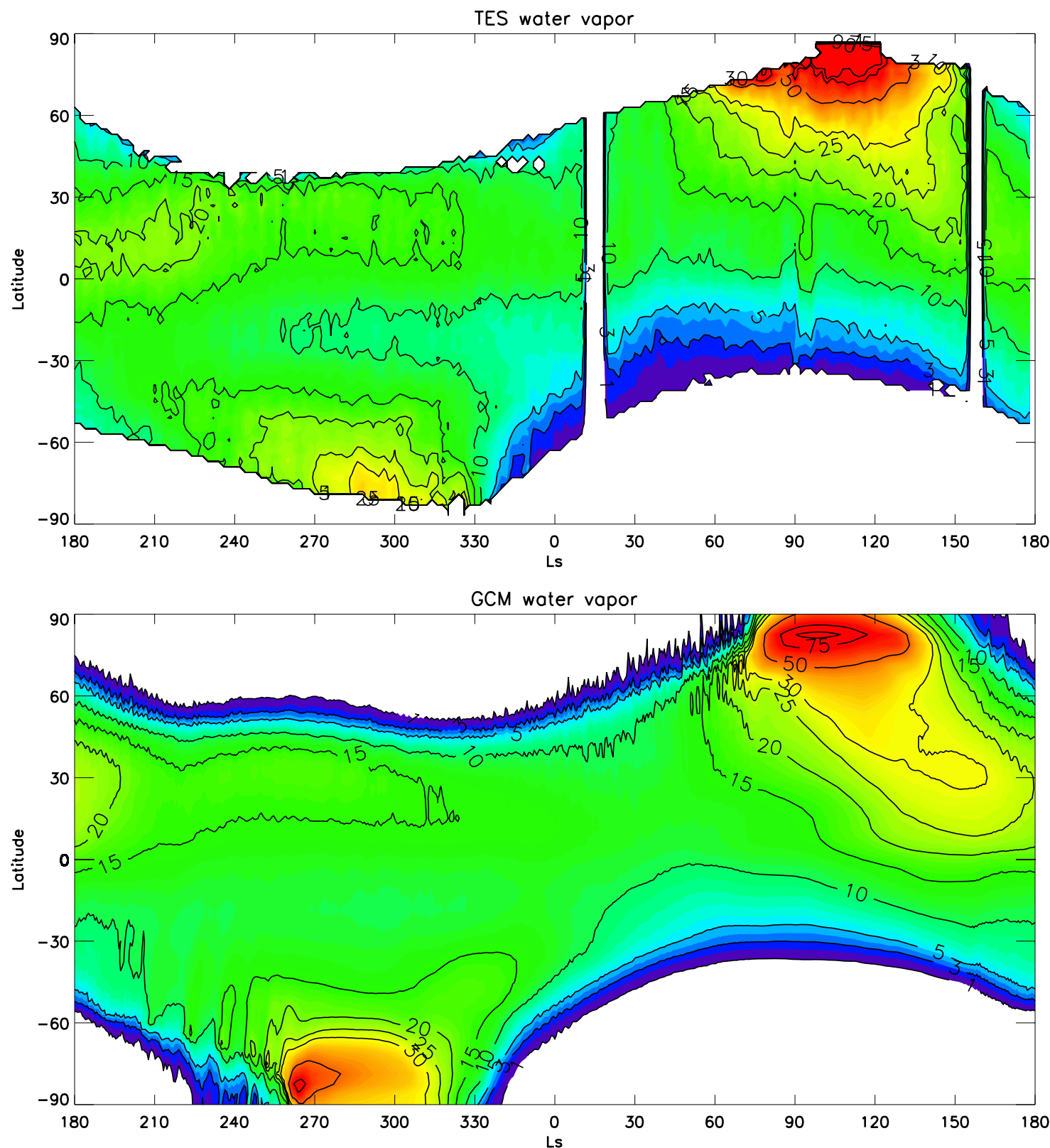

Figure 2. Seasonal and latitudinal distribution of water vapor in pr. $\mu \mathrm{m}$ (top) as derived from TES observations [Smith, 2004] and (bottom) as given by our model for the sixth year of simulation.

wave activity (though the nature of the waves was not discussed by Richardson and Wilson [2002]) could be responsible for the intense horizontal mixing of water vapor across the cap edge, and its ultimate deposition between the cap edge and the pole. As the cap recedes, the seasonal water ice frost alternately sublimes and then recondenses within poleward warm fronts and equatorial cold fronts. In this way, most of the water cold-trapped during winter is released when the cap recedes, transported poleward, and then redeposited onto the cap. The annual water budget in the arctic is thus closed during the final stage of the cap recession when water is carried into the vicinity of the north pole.

[44] The most convincing evidence supporting such a return of water to the pole in a "quasi-solid state" [Richardson and Wilson, 2002] has been recently presented by Titus and Kieffer [2003]. Measurements of surface albedo combined with frost grain estimates clearly suggest that part of the water vapor released by the retreating cap is redeposited as frost inside the cap. Consequently, the cycling of water associated with the waxing and waning of the seasonal $\mathrm{CO}_{2}$ cap plays a crucial role in the Martian hydrological cycle.

[45] All in all, the mechanism by which the water cycle achieves a putative steady-state mainly involves horizontal mixing processes between the arctic region and midlatitudes. All year long, horizontal mixing tends to relax latitudinal gradients of humidity between the north pole, where local vapor concentrations are seasonally controlled by insolation variation, and middle to low latitudes where water abundances are "history dependent" [Richardson and Wilson, 2002]. To summarize all the ideas introduced so far, a schematic sketch of the water cycle is presented in Figure 3. 


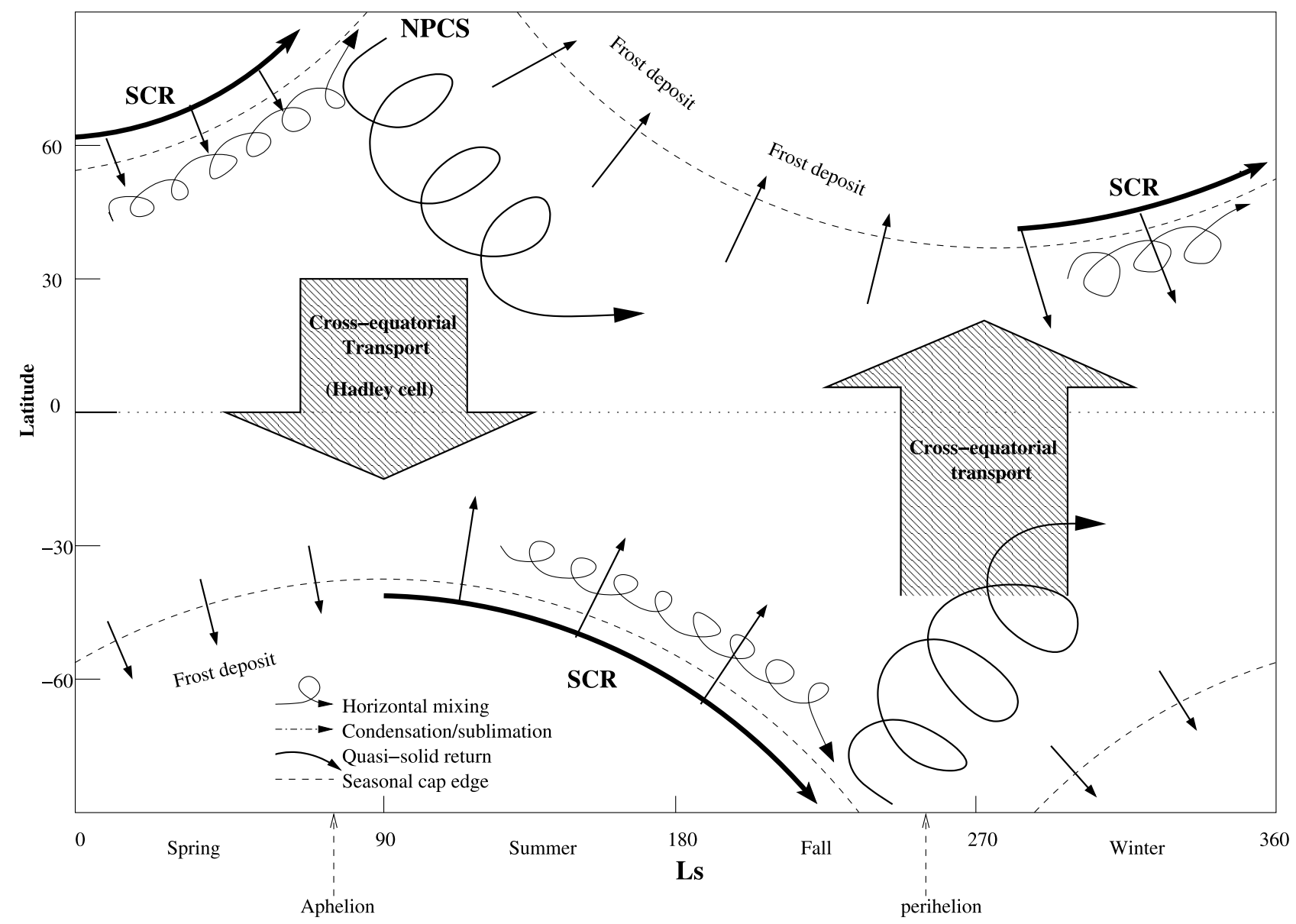

Figure 3. Chart describing the principal events affecting the Martian water cycle over the course of a year. NPCS stands for North Polar Cap Sublimation; SCR stands for Seasonal Cap Recession.

[46] Another comparison of TES data with the model simulation is given in Figure 4. Here, the quantity of interest is the integrated mass of water vapor in each hemisphere as a function of solar longitude $\left(\mathrm{L}_{s}\right)$. In the northern hemisphere, the simulated evolution of water content closely follows the data points until approximately $\mathrm{L}_{s}=180^{\circ}$, where model and observations start to deviate. The modeled decrease of humidity appears steeper than observed in midsummer and fall. In contrast, the predicted increase of humidity in the southern hemisphere at the same season is markedly lower than indicated by TES. These two elements could have the same origin. On the basis of the observations, Smith [2002] suggested that the overturning circulation conveys a significant flow of water from south to north, potentially balancing the mass of water vapor cold-trapped by the seasonal cap. It is therefore likely that our model fails at reproducing the actual release of water vapor in the southern hemisphere as the $\mathrm{CO}_{2}$ cap recedes. On the other hand, both model and observations suggest a much slower variation of humidity in the north during the time frame bracketing perihelion, supporting the idea of a crossequatorial flow of water out of the south. Despite an overall good match of the TES observations in the north, the variation of humidity in the southern hemisphere is not as well reproduced. The model does not accurately simulate the rapid decrease in moisture that begins around $\mathrm{L}_{s}=300^{\circ}$ and continues until midspring.

\subsection{Cloud Predictions}

\subsubsection{Main Features}

[47] Figure 5 presents the seasonal evolution of the cloud mass fraction in the total inventory of atmospheric water.

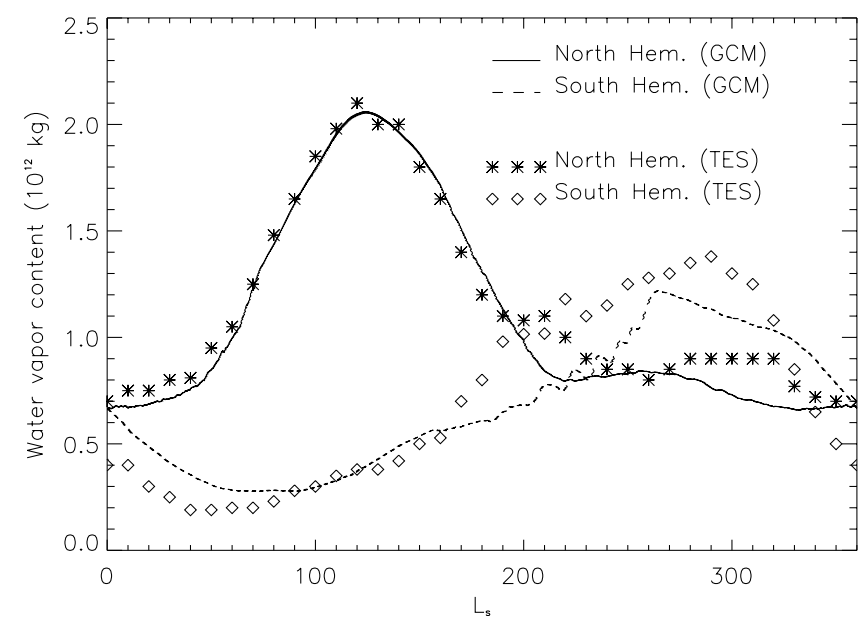

Figure 4. Comparison between the seasonal evolution of the integrated mass of water vapor in each hemisphere produced by our model and by that inferred from TES data [Smith, 2002]. 
This figure shows the strong forcing exerted by Martian orbit on the seasonal behavior of water ice clouds. On a global average, cloud fraction fluctuates around a value of $9 \%$ and follows a seasonal variation imparted by that of solar insolation.

[48] However, if this statement was completely true, we should observe the global ratio peaking near aphelion. This is not the case as this ratio reaches a maximum near the vernal equinox $\left(\mathrm{L}_{s} \sim 0^{\circ}\right)$, as does that of the northern hemisphere.

[49] Near aphelion, cloud fraction is predicted to be at its maximum in the southern hemisphere. This coincides with the cooler atmosphere of the equatorial regions (driven by a lower dust loading, a decrease in insolation and a strong adiabatic cooling in the upwelling branch of the Hadley cell), forcing the hygropause to be confined to lower layers. This, in turn, increases the water mass potential for condensation and therefore favors cloud formation. Interestingly, the cloud ratio in the south is double peaked, with a secondary, but very sharp peak occurring near northern fall equinox. This pattern will be discussed in the next section. However, the double-peaked behavior can be found in any of the three curves displayed in Figure 5.

[50] In addition to the enhancement of solar flux between aphelion and perihelion, the dust loading also increases during the same period (this is the MGS dust scenario). This represents the transition between a clear aphelion atmosphere and a more dusty perihelion atmosphere that has been observed for decades. Accordingly, the mean level of the hygropause in the equatorial region is shifted from an altitude of roughly $10 \mathrm{~km}$ around $\mathrm{L}_{s}=90^{\circ}$ to a height of about $40 \mathrm{~km}$ near $\mathrm{L}_{s}=250^{\circ}$ (not shown). This agrees well with what Smith [2002] inferred from the data. It is therefore not surprising to note from Figure 5 that southern summer corresponds to a minimum in the global cloud ratio, a statement that is more in line with the idea of an orbitally driven cloud cycle. More surprising is the fact that it also coincides with a period of relatively low cloud mass in the north, where the polar vortex almost achieves its maximum extent.

\subsubsection{Distribution Versus Latitude}

[51] With their broad absorption feature centered around a wavelength of $12 \mu \mathrm{m}$, water ice clouds have been detected by most of the infrared instruments that have operated on board Mariner 9, Viking and MGS orbiters. However, it is only recently that these data have been compiled in order to yield the first monitoring of water ice clouds for more than one Martian year and at most latitudes. Tamppari et al. [2000] used the Viking Infrared Thermal Mapper IRTM to derive brightness temperature contrasts generated by cloud absorption. Their results reveal a prominent presence of clouds in the equatorial band around northern summer solstice and some evidence of clouds forming in the fall/ winter hemispheres. These authors complemented their first study in a later paper addressing the question of the cloud diurnal cycle as seen by Viking [Tamppari et al., 2003]. Despite their great scientific value, these data have restricted spatial coverage in comparison to the more recent TES measurements. The sun-synchronous, nearly polar orbit of MGS around Mars allowed for observations of the same point at fixed local time (2AM and 2PM). However, aerosol opacity retrievals are restricted to areas with surface tem-



Figure 5. Seasonal change of the water cloud reservoir expressed as a fraction of the total atmospheric reservoir of water (cloud + vapor).

perature greater than $220 \mathrm{~K}$, therefore excluding nighttime dust and cloud opacity measurements as well as observations over the cold surface of both seasonal caps.

[52] Since the beginning of the MGS mission, which has now entered an extended phase, TES has been able to collect cloud data for almost three Martian years [Smith, 2004]. One of the most striking features concerning water ice clouds is the relative lack of interannual variability, at least in the observable zones, which might be related to their quasi-absence during seasons where large and variable dust storms are most likely to occur. Liu et al. [2003] address this issue in their compilation of atmospheric data collected since the Mariner 9 mission. One of their conclusions emphasizes the quite repeatable meteorological pattern of the aphelion season, regardless of the spacecraft era.

[53] In Figure 6 (top graph), we show the seasonal and latitudinal distribution of water ice clouds as observed by TES. As explained by Smith [2004], the opacity derived from TES measurements does not represent the total opacity of the clouds. By neglecting infrared scattering in their radiative transfer model, the TES retrievals displayed in Figure 6 represent extinction due to cloud absorption/ emission. Smith [2004] recommends applying a scaling factor roughly equal to 1.5 to determine the true extinction. We also show for comparison the cloud distribution predicted by our model (bottom graph). The absorption opacity has been computed from

$$
\tau=\frac{3 Q_{a b s} M_{c}}{4 \rho_{i} r_{c}}
$$

where $Q_{a b s}$ is the absorption efficiency of water ice at $12 \mu \mathrm{m}$ $(\sim 1), M_{c}$ the integrated cloud mass predicted by the model $\left(\mathrm{kg} . \mathrm{m}^{-2}\right)$ and $r_{c}$ the predicted size of the particles (in meters).

[54] In general, our model captures most of the observed cloud geographical and seasonal distribution. During the aphelion season, water sublimed from the north polar cap reaches the tropics. Once incorporated in the upwelling branch of the solsticial Hadley cell, moist air masses are 

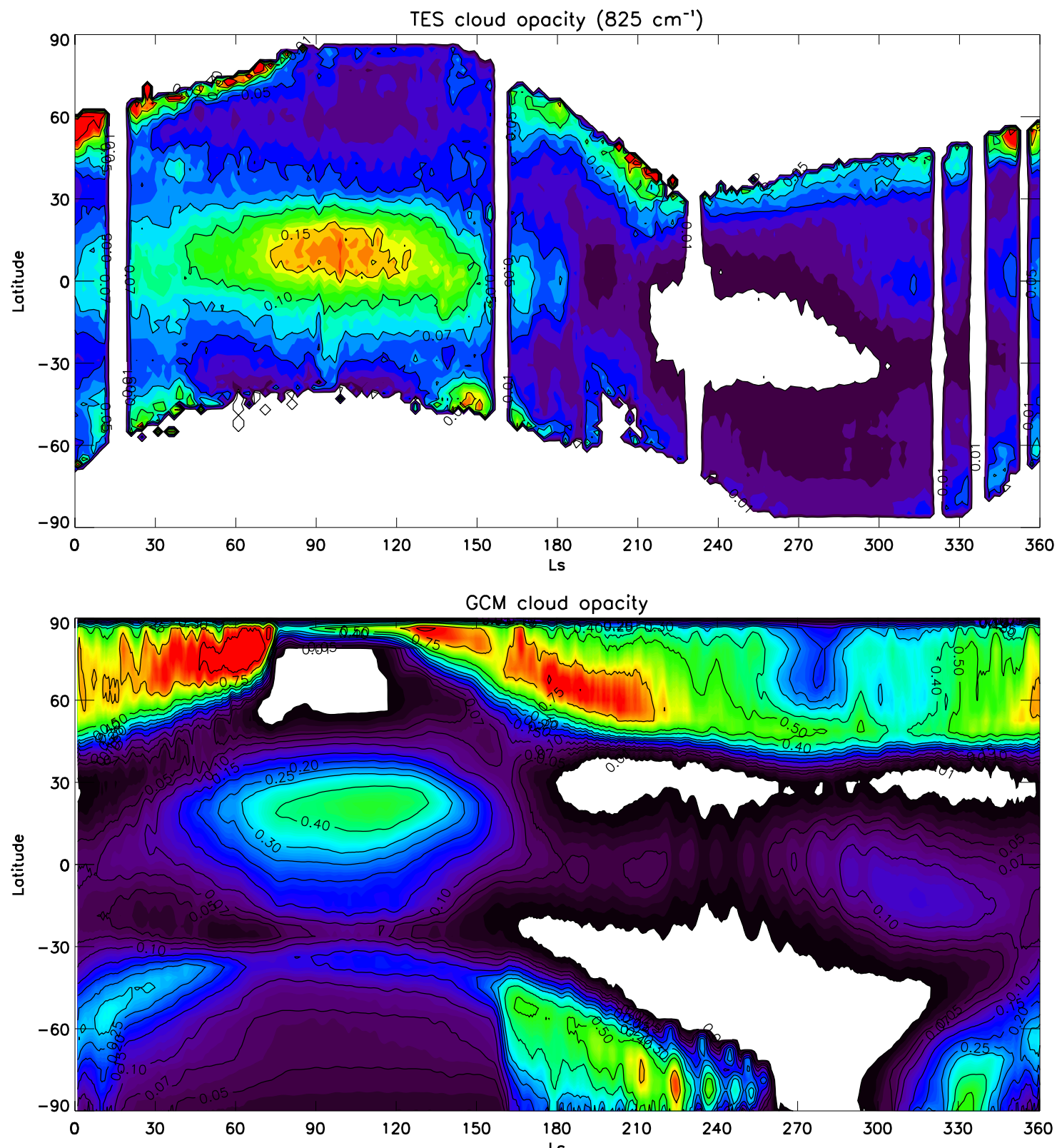

Figure 6. Seasonal and latitudinal distribution of water cloud opacity (top) as derived from TES observations [Smith, 2004] and (bottom) as given by our model. Model data have been sampled to 2PM local time to remove the potential bias induced by cloud diurnal variability and to allow comparison with observations.

adiabatically cooled, and in most cases become saturated with respect to water during their ascent. The combination of these factors gives rise to the so-called "equatorial cloud belt" (ECB hereafter) [Clancy et al., 1996; Wolff et al., 1999], which appears as the main feature of the cloud cycle seen by TES. It is generally believed that the latitudinal extent of the ECB coincides with the theoretical boundaries of the overturning circulation. Indeed, both model and TES data suggest the heart of the ECB is offset somewhat north of the equator, whereas the thickest portion lies between $30^{\circ} \mathrm{N}$ and $10^{\circ} \mathrm{S}$. Again, model and observations also agree on the timing of formation and decline of the ECB. In fact, the decay of the belt is clearly related to an increase of temperature in the equatorial region and to the transition toward a sluggish equinox circulation. Indeed, the belt declines shortly after the northern summer solstice, despite an increasing level of humidity in the same region.

[55] On the other hand, our model substantially overestimates cloud opacities ( 0.4 versus 0.2 at the peak of ECB). Many reasons can be invoked to explain this. Foremost among them is the issue of the thermal behavior at the cloud level. While neglecting cloud radiative feedbacks and dust-cloud interactions, we omit an important component of the actual synergy between microphysics and radiation. Also, we already mentioned that our simple model is, in principle, likely to overestimate cloud mass compared to a reference cloud model. Other possibilities might involve a more rigorous choice of our $Q_{a b s}$ value that 
would vary with particle size, and some uncertainties related to the retrieval of cloud opacities from spacecraft data. For instance, Liu et al. [2003] shows how sensitive this quantity is to the assumption of the cloud particle size, with opacities changing by more than a factor 2 for a likely range of particle radii. Finally, we should mention that the vertical resolution employed by the GCM is not fully adequate to the representation of the cloud vertical structure. Being nonlinearly dependent on temperature (by virtue of the vapor pressure law), clouds require an accurate resolution of the thermal profile, at least in their atmospheric portion. A simple experiment conducted with our reference cloud scheme suggests that the use of GCMs vertical resolution generates a cloud opacity roughly two times larger than a more refined vertical grid (with a $\sim 2 \mathrm{~km}$ spacing). This factor 2 cannot be representative of all possible cases, as the irregular GCMs grid spacing increases with height. It is, however, conceivable that a lack of vertical resolution near the hygropause can affect the determination of the condensation zone and artificially change the predicted cloud depth.

[56] Inspection of Figure 6 gives some insight into the behavior of clouds in the polar winter. As previously mentioned, TES is unable to confidently retrieve aerosol opacities above the $\mathrm{CO} 2$ seasonal caps. Comparison of the two graphs shows that this limitation potentially excludes a significant part of the cloud annual cycle, i.e., the Polar Hoods. Our model suggests that some of the thickest Martian clouds (on a zonally-averaged sense) could actually be found in the Polar Hoods. TES data near the seasonal cap edges support this hypothesis (especially in the north during springtime). According to the model, the vertical structure of the Polar Hood clouds resembles that of fogs. They extend from the surface up to about a scale height.

\subsubsection{Hemispheric Asymmetries of the Polar Hoods}

[57] The intensity of the north Polar Hood markedly contrasts with that of the southern hemisphere, especially during their respective seasons of expansion and recession. This difference is generally attributed to the fact that the southern winter hemisphere is much dryer than its northern counterpart. However, the intensity of the hoods also depends on the efficiency in water vapor supply to the polar regions, which is a strong function of the eddy activity. In their paper discussing polar processes, Pollack et al. [1990] showed a prominent asymmetry in transient eddy activity between the two hemispheres during their respective fall, winter and spring seasons. The southern winter hemisphere exhibits a much weaker activity, that Zurek et al. [1992] suggested was due to a more stabilizing configuration of topography in the southern high latitudes.

[58] In order to quantify the meridional water vapor flux due to stationary and transient eddies, we use the decomposition given by Peixoto and Oort [1992], where water transport is broken down into several circulation components. It is straightforward to demonstrate that the total meridional transport of a tracer species includes the contribution of the mean meridional circulation, transient eddies, and stationary waves.

[59] Following this approach, we express the total transport of water vapor at a given latitude and at a given height as $[\overline{q v}]$, where the $\left(^{-}\right)$symbol denotes the time average and [] symbols denotes the zonal mean of the product $q v, q$ being the mass mixing ratio of water and $\mathrm{v}$ the meridional wind. According to Peixoto and Oort [1992], [ $\bar{q} v]$ can be written as

$$
[\bar{q} v]=[\bar{q}][\bar{v}]+\left[q^{\top} v^{\prime}\right]+\left[\bar{q}^{*} \bar{v}^{*}\right]
$$

with the prime symbol expressing the departure from the time average $\left(q^{\prime}=q-\bar{q}\right.$ and $\left.v^{\prime}=v-\bar{v}\right)$ and the star symbol being related to the departure from the zonal average $\left(q^{*}=\right.$ $q-[q]$ and $\left.v^{*}=v-[v]\right)$.

[60] Thus total water transport $[\overline{q v}]$ is the sum of the mean meridional circulation component $[\bar{q}][\bar{v}]$, that of transient eddies $\left[q^{\top} v^{\prime}\right]$ and that of nontraveling waves $\left[\bar{q}^{*} \bar{v}^{*}\right]$. Equation (5) can be integrated over height to yield

$$
\int_{0}^{p_{s}}[\bar{q} v] \frac{d p}{g}=\int_{0}^{p_{s}}[\bar{q}][\bar{v}] \frac{d p}{g}+\int_{0}^{p_{s}}\left[q^{\top} v^{\prime}\right] \frac{d p}{g}+\int_{0}^{p_{s}}\left[\bar{q}^{*} \bar{v}^{*}\right] \frac{d p}{g}
$$

[61] Given that $q$ is the sum of both atmospheric water vapor and water ice (clouds), equations (5) and (6) can be further decomposed into the respective contributions of vapor and clouds. Furthermore, in order to remove any signal associated with the diurnal cycle, $q$ ' has been filtered in time to retain only those periods longer than a day.

[62] Figure 7 illustrates the behavior of the eddy meridional transport of water vapor during the spring formation season of each Polar Hood. A steady prominence of the northern eddy moisture fluxes is readily apparent. According to Figure 7, both transient and stationary eddies play a major role in supplying water to the Polar Hoods. In the southern hemisphere, the Hellas Basin generates a strong current along its southeastern rim, leading to a significant poleward flow of moisture. The same pattern appears along the southeastern rim of the Argyre basin. In the northern hemisphere, the Tharsis rise in midlatitudes creates a similar wind configuration and therefore forces an important poleward transport of water vapor. The eddy fluxes in the northern hemisphere are stronger than those in the southern hemisphere during the hood forming season. This asymmetry is the primary reason the north Polar Hood is more pronounced than its southern hemisphere counterpart.

\subsubsection{Seasonal Evolution of the Polar Hood}

[63] Despite their differences in terms of cloud mass, the two polar hoods exhibit comparable behavior. Both increase during the onset of the polar vortex. Concentrations of water vapor are still very high at the end of summer in both hemispheres, since maximum atmospheric holding capacity is achieved at these seasons. As the temperature decreases, so does holding capacity, and clouds start to form. Meanwhile, eddies strengthen their activity and supply water vapor from the moist equatorial regions. Around the solstices, the Polar Hoods almost disappear and are essentially confined to the seasonal cap edge. In fact, this behavior is the result of a significant reduction in wave activity in the winter hemisphere. As shown in Figure 8, the eddy flux of clouds becomes negligible around the solstices in the winter hemisphere. This reflects a lack of horizontal mixing of water vapor (not shown) in the same region for the exact same reason. Such a decrease in horizontal mixing is not well understood, but appears to be a feature common to other GCMs (R. M. Haberle et al., The NASA Ames 

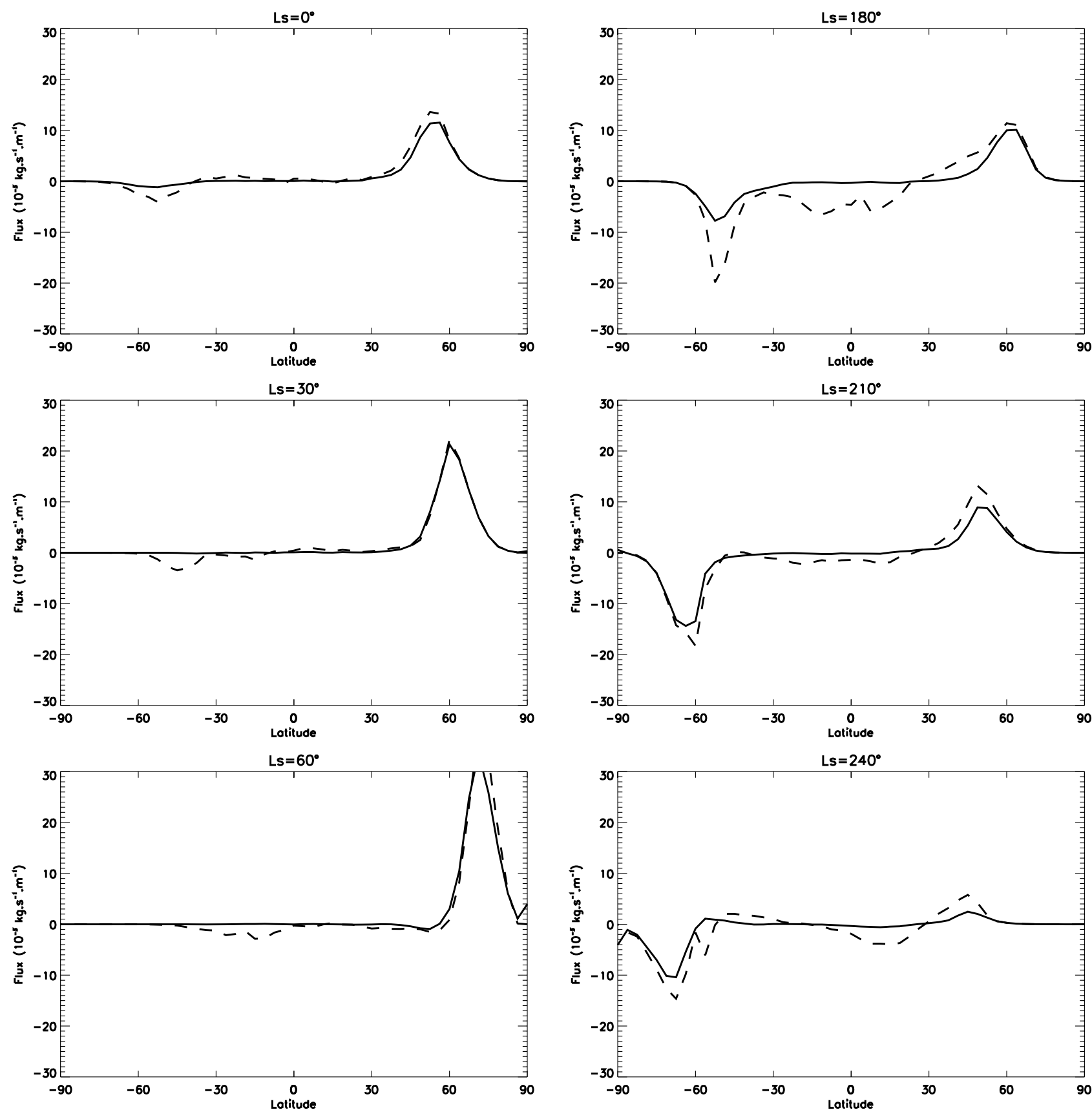

Figure 7. Vertically integrated water vapor flux due to meridional wind fluctuations as a function of latitude. Each plot denotes a different season. The time frame bracketed between $\mathrm{L}_{s}=0^{\circ}$ and $\mathrm{L}_{s}=60^{\circ}$ corresponds to the formation (decay) of the North (South) Polar Hood, and the time frame bracketed between $\mathrm{L}_{s}=180^{\circ}$ and $\mathrm{L}_{s}=240^{\circ}$ gives the opposite situation. The solid line indicates water vapor transport made by transient eddies, and the dashed line is for stationary eddies. Southward transport is counted negative.

Martian General Circulation Model: Updates and comparison with observations, manuscript in preparation, 2004; hereinafter referred to as Haberle et al., manuscript in preparation, 2004).

[64] In the postsolsticial season, the Polar Hoods return to a period of intense formation, which correlates with the seasonal cap recession. In the south, clouds are abruptly triggered around $\mathrm{L}_{s} \sim 160^{\circ}$. Preceding this event, clouds were mostly located over the border of the cap, whereas after they extend all the way to the south pole. This explains the secondary peak in southern hemisphere cloud fraction displayed in Figure 5. A similar increase occurs for the north Polar Hood $\left(\mathrm{L}_{s} \sim 300^{\circ}\right)$, though it is much more gradual. Although no firm conclusions can be drawn from the TES cloud map (Figure 6), the seasonal changes seen by TES in the cap edge Polar Hoods are comparable to those given by the model. These changes can be sensed by examining the opacity variation for the clouds evolving 



Figure 8. Same presentation as in Figure 7, except for water ice clouds.

near the border of the unobserved zones. Our model indicates that a sudden growth of both stationary and transient waves near the equinoxes explains the reintensification of the Polar Hoods (a similar trend is noted by Haberle et al. (manuscript in preparation, 2004)).

[65] Interestingly, Figure 8 displays a change in direction of the cloud flux in the region between the midlatitudes and the pole. For instance, there is transition around $60^{\circ} \mathrm{S}$ at $\mathrm{L}_{s}=$ $180^{\circ}$ (the same transition occurs near $60^{\circ} \mathrm{N}$ ). South of the boundary, the cloud flux is oriented toward the pole (explaining the poleward extension of the Polar Hoods of Figure 6), whereas clouds propagate equatorward north of the boundary. This is the consequence of the nature of eddies which tends to relax the latitudinal gradient of water ice clouds. The gradient is forced locally by a cloud formation peaking near the edge of the polar vortex where wet air masses cool intensively as they migrate poleward.

\subsubsection{Cloud Microphysical Properties}

[66] Through sedimentation, clouds can affect the vertical distribution of water vapor [Michelangeli et al., 1993]. When convolving this effect with wind vertical structure, clouds can consequently modulate the horizontal transport of water as a whole. However, the magnitude of this process will depend uniquely on the cloud particle size.

[67] Figure 9 gives the cloud particle radius distribution with season and latitude. The cloud particle radius given by the model is found to vary between 1 and $10 \mu \mathrm{m}$. Previous GCM simulations of the water cycle [Richardson and Wilson, 2002; Richardson et al., 2002] assumed a constant particle size for sedimentation $(2$ or $15 \mu \mathrm{m})$. Our first results are therefore in stark contrast with this approximation and might provide some insights on the necessity for these authors to use an unrealistic particle size to match water cycle observations.

[68] Our results agree particularly well with the estimates of Clancy et al. [2003], who define two categories of clouds: Type I clouds which are mostly found in the southern hemisphere between $\mathrm{L}_{s}=30$ and $110^{\circ}$ and which have particle effective radii in the $1-2 \mu \mathrm{m}$ range; and Type II clouds which occur almost exclusively in the ECB and which have radii in the 3 to $5 \mu \mathrm{m}$ range. Figure 9 shows that the model-predicted sizes are consistent with Type I clouds for the same period and locations. Thus, compared to the available observations, the model appears quite capable of 


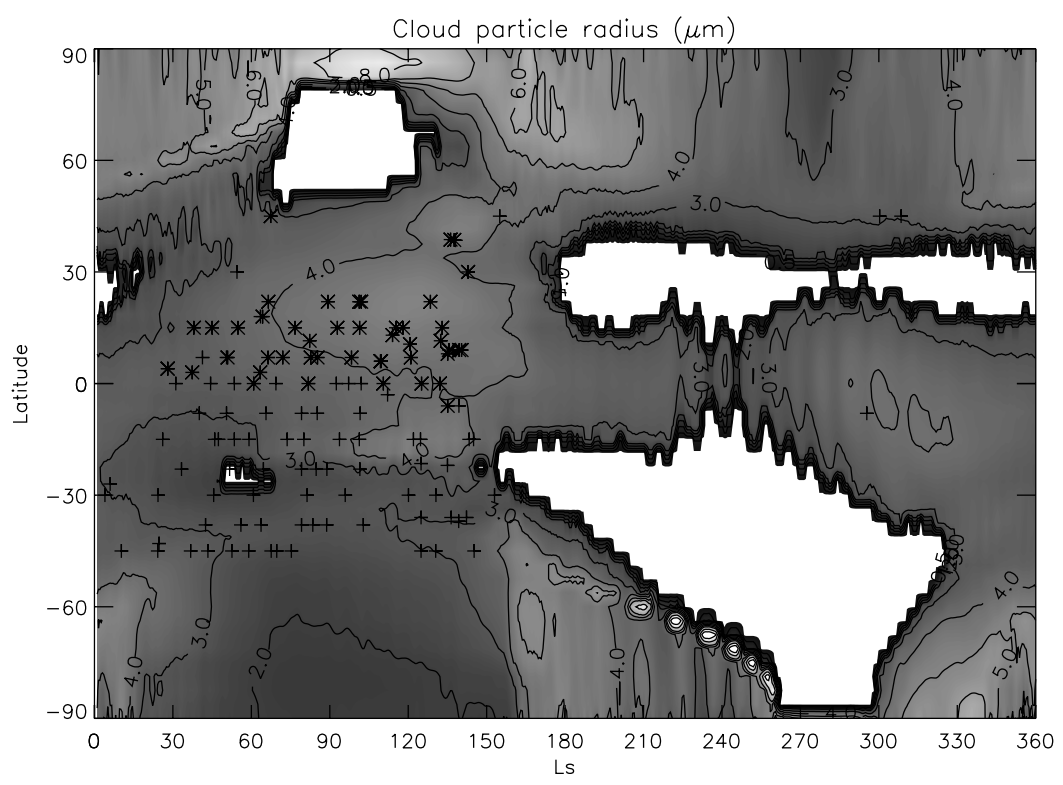

Figure 9. Effective cloud particle size predicted by the MGCM as a function of season and latitude. We superimposed the estimates made by Clancy et al. [2003] from TES Emission Phase Function measurements (data kindly provided by R. T. Clancy). Crosses are for type 1 clouds, having 1 to $2 \mu \mathrm{m}$ particle radius, and stars are for type 2 cloud particles with sizes ranging from 3 to $5 \mu \mathrm{m}$. Although model predictions do not perfectly fit Clancy's estimates, the latitudinal and seasonal trends are nevertheless respected.

simulating not only the distribution of clouds but their particle sizes as well.

[69] Furthermore, the MGCM indicates substantial seasonal variations of particle sizes in both polar regions. Polar Hood clouds exhibit markedly larger particles during their early stage of formation and during the final stages of seasonal cap retreat. In these cases, it is not unusual to find particles larger than $5 \mu \mathrm{m}$. In our cloud scheme, particle size is mainly determined by the amount of condensed species. Since the Polar Hoods are predicted to be thicker during the same periods, cloud particle sizes will increase accordingly. 3.2.6. Northern Summer Clouds in the Polar Region

[70] Probably the most intriguing feature of Figure 9 is the presence of large cloud particles above the north pole during spring and summer. A polar projection of the arctic region during summer solstice is given in Figure 10. As water vapor sublimates from the north polar cap, the lowest atmospheric layers become quickly saturated and allow clouds to form near the ground (typically in the first 5 kilometers).

[71] This "Summer Polar Hood" is predicted to be thick (as much as the ECB) and features particle sizes on the order of $10 \mu \mathrm{m}$. In their study of the water ice cloud radiative feedback, Colaprete and Toon [2000] make the case of ground fog formation and report particle sizes as large as $15 \mu \mathrm{m}$, suggesting that at low altitudes, much larger particle radii can be expected (near the ground, the denser atmosphere allows particles to be kept aloft longer, a timescale of several days for $10 \mu \mathrm{m}$ particles is typical). Whether these clouds do occur in reality is a difficult issue to address. Their proximity to the ground implies very low brightness temperature contrasts if probed by an infrared instrument like TES. Also, the detection of these hazes by a camera (MOC) is made difficult by the underlying bright surface of the cap. MOLA data, however, support their presence [Neumann et al., 2003]. To quote these authors: "Daytime clouds are evident at latitudes $>65^{\circ}$, forming a band that recedes to the permanent cap as summer approaches, remaining until $\mathrm{L}_{s}=160^{\circ}$."

[72] Despite their likelihood, such clouds result from the combination of complicated processes, involving ground ice sublimation, cloud microphysics and planetary boundary layer (PBL) dynamics. As ground ice sublimation is critically dependent on surface properties, the actual spatial heterogeneity of the cap (with the succession of bright and dark deposits at the kilometer scale) should challenge the predictions made by our model. Taken together, these facts suggest that these clouds should be seen as localized events, possibly sub-grid scale cumuli developing in the PBL, rather than a diffuse low-lying haze.

[73] Nonetheless, their presence above the cap in summer is of prime importance (at least for the model) for redistributing water vapor vertically and consequently throttling the sublimation rate. As stated previously, cloud sedimentation increases the confinement of water in the lower atmosphere and in some cases can lead to precipitation down to the surface. During the sublimation season of the polar cap, the presence of these low clouds balances the upward flux of the subliming water by a substantial amount of precipitation and by restraining the propagation of water at higher levels where horizontal advection is more efficient. This way, clouds partly control the amount of water that is effectively extracted from the cap in spring and summer.

\subsubsection{Clouds Related to Topography}

[74] Several images of the ECB show that its longitudinal structure is apparently forced by topography, with the thickest clouds forming along the flanks of the major volcanoes [James et al., 1996; Wang and Ingersoll, 2002]. 

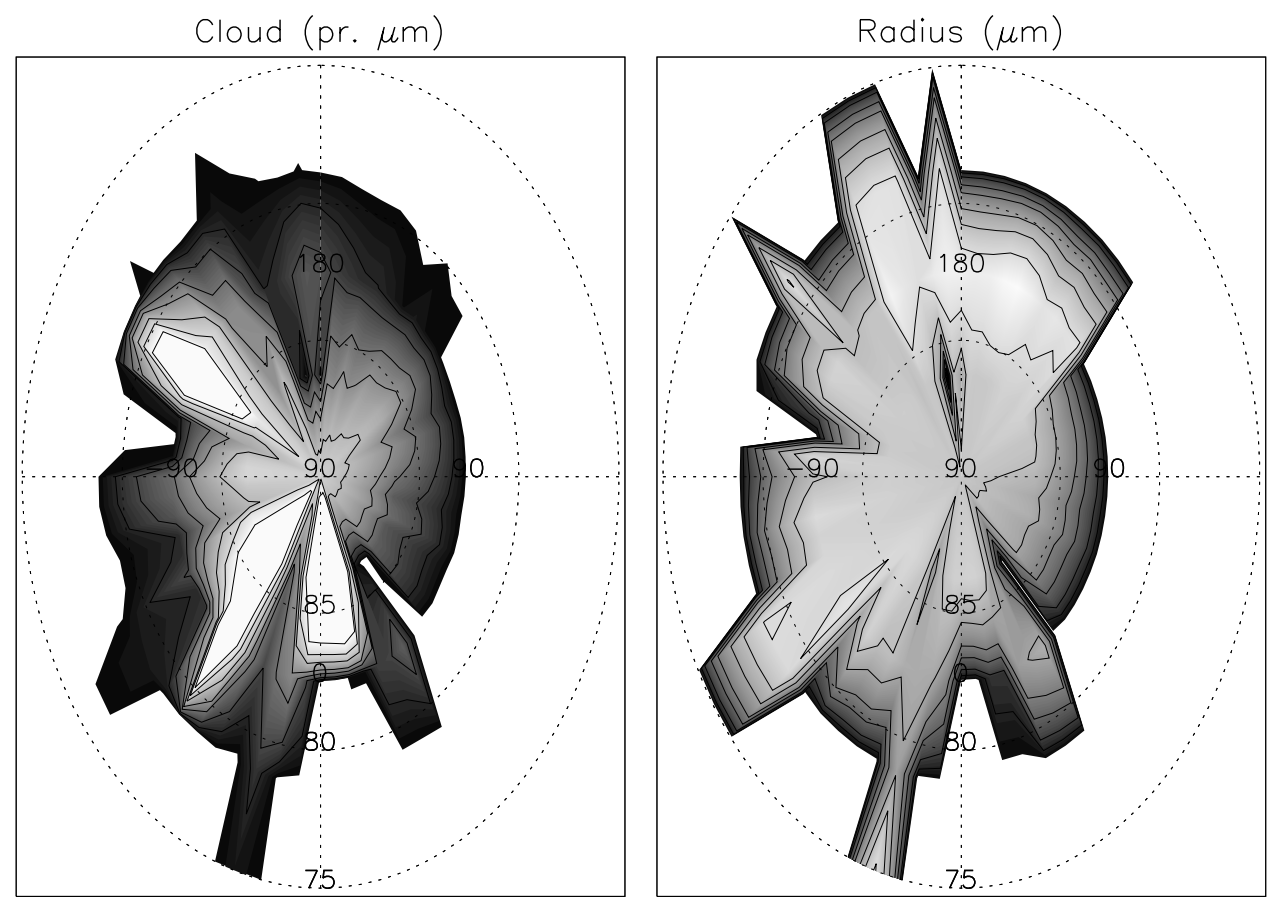

Figure 10. Stereographic projections of the north pole at $\mathrm{L}_{s}=90^{\circ}$ as produced by the model (2PM local time). On the left are reported the integrated cloud mass values (levels set at 0.5, 1, 2, 3, 5, 7, and 10 pr. $\mu \mathrm{m}$; brighter areas indicate increasing cloud mass). The right graph gives the effective radius of the cloud particles (same levels as above except in $\mu \mathrm{m}$ ). Particles found in this region at this season are the largest of the whole annual cloud cycle.

It is generally assumed that such orographic clouds form during the day when moisture carried in upslope flows, is cooled adiabatically and forms thick condensate clouds with opacities higher than 0.5 in the visible [James et al., 1996].

[75] Recently, Benson et al. [2003] documented topographic clouds, using the global maps obtained by MOC. They specifically tracked some of the major topographic features (Tharsis Montes, Olympus Mons, Alba Patera, etc.) over the course of a Martian year to establish their respective seasonal trends. One of their most interesting findings concerns the double peaked behavior exhibited by Alba Patera, where clouds peak at $\mathrm{L}_{s}=60$ and 140 and reach a minimum in-between. In contrast, all the volcanoes of the Tharsis plateau showed rather continuous progression and decay around $\mathrm{L}_{s}=90^{\circ}$ (see Figure 11).

[76] This Alba Patera "anomaly" has been investigated with our model, for which results have been reported in Figure 12. Though less marked than those observed, we also find that this region exhibits two successive peaks of cloud formation, separated by $60^{\circ}$ in solar longitude. The first peak occurs at $\mathrm{L}_{s}=60^{\circ}$ and is followed by a slight decrease to a level that will remain constant until $\mathrm{L}_{s}=90^{\circ}$. By plotting the corresponding water vapor amount at this location, we can see a clear correlation between the second cloud peak and the humidity level. This indicates that the maximum reached at $\mathrm{L}_{s}=140^{\circ}$ is a consequence of an increase in humidity, which is supplied by the subliming north polar cap. The first peak at $\mathrm{L}_{s}=60^{\circ}$, however, is less amenable to such a straightforward interpretation. Even though it also corresponds to a slight increase in water vapor, it also appears marked by some large, high-frequency (on the order of a couple of days) oscillations, which could be interpreted as intensified wave activity in the region. Prior to the permanent cap sublimation season, the water vapor increase can only be caused by the retreat of the seasonal cap and the subsequent evaporation of its water ice content. Baroclinic eddies accompany the cap recession and can explain the enhancement of cloudiness in this region. Then, as the cap retreats further, the crater becomes too far from the cap edge to be influenced by the eddies, thus explaining why clouds tend to decrease shortly after $\mathrm{L}_{s}=$ $60^{\circ}$. Figure 13 illustrates clearly the behavior of cloud formation via transient eddies in the Alba Patera region. This figure shows that the Tharsis volcanoes are essentially unaffected by wave activity.

[77] The comparison with the seasonal pattern of Ascraeus Mons (bottom graph of Figure 12) shows distinctive cloud behavior. The Tharsis volcano follows the usual trend of the $\mathrm{ECB}$, and is therefore driven by both insolation and large-scale flows. Arguably, this difference in seasonal cloud activity is the consequence of the mean circulation. Whereas Ascraeus Mons $\left(10^{\circ} \mathrm{N}, 105^{\circ} \mathrm{W}\right)$ is located right in the upwelling zone of the Hadley cell, the region of the Alba Patera crater $\left(40^{\circ} \mathrm{N}, 110^{\circ} \mathrm{W}\right)$ is even more northward than the northernmost boundary of the cell and should therefore be too far to be affected by it (see Forget et al. [1999] for some cross sections of the meridional stream function).

\subsubsection{Polar Hood in the Northern Hemisphere}

[78] In this part, we will focus on two particular manifestations of the north Polar Hood that have been discussed in previous papers.

\subsubsection{Northern Fall Streak Clouds}

[79] In their article detailing the observations of water ice clouds made by MOC, Wang and Ingersoll [2002] describe 

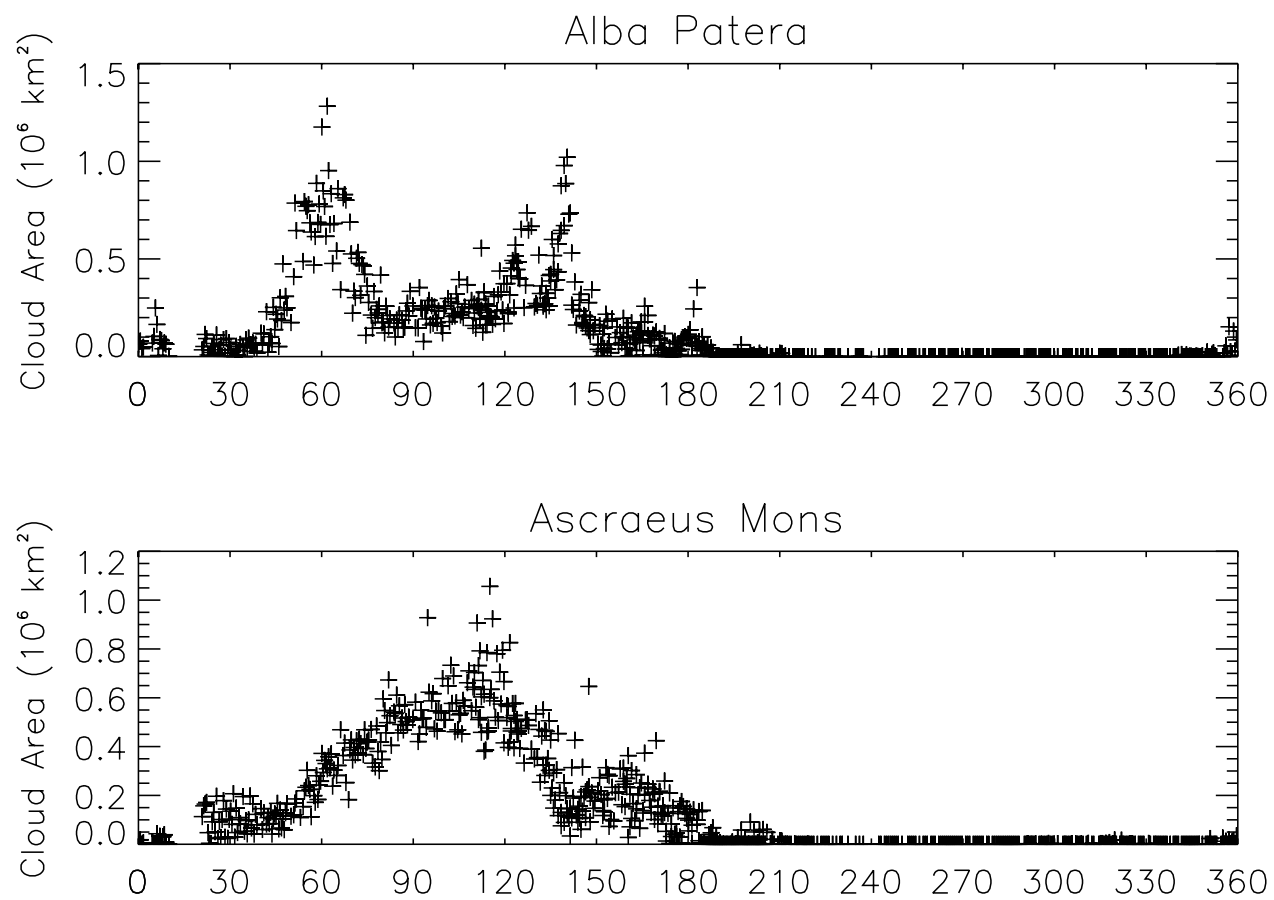

Figure 11. (top) Cloud area versus $\mathrm{L}_{s}$ at Alba Patera as inferred by Benson et al. [2003] from MOC images. (bottom) Same as above but for Ascraeus Mons. Both panels have been extracted from Benson et al. [2003] (their Figures 4b and 6).

a particular occurrence of the north Polar Hood: the north polar streak clouds. The latter present the most characteristic structure of all the clouds evolving in the Polar Hood. Usually observed between midnorthern fall and midnorthern winter, they appear as long spirals extending from the cap edge up to the north pole. As stated by Wang and Ingersoll [2002], their counterclockwise orientation seems indicative of low-level winds converging poleward. As shown by Figure 14, such clouds are effectively simulated by the model. Whereas this figure mostly exhibits the
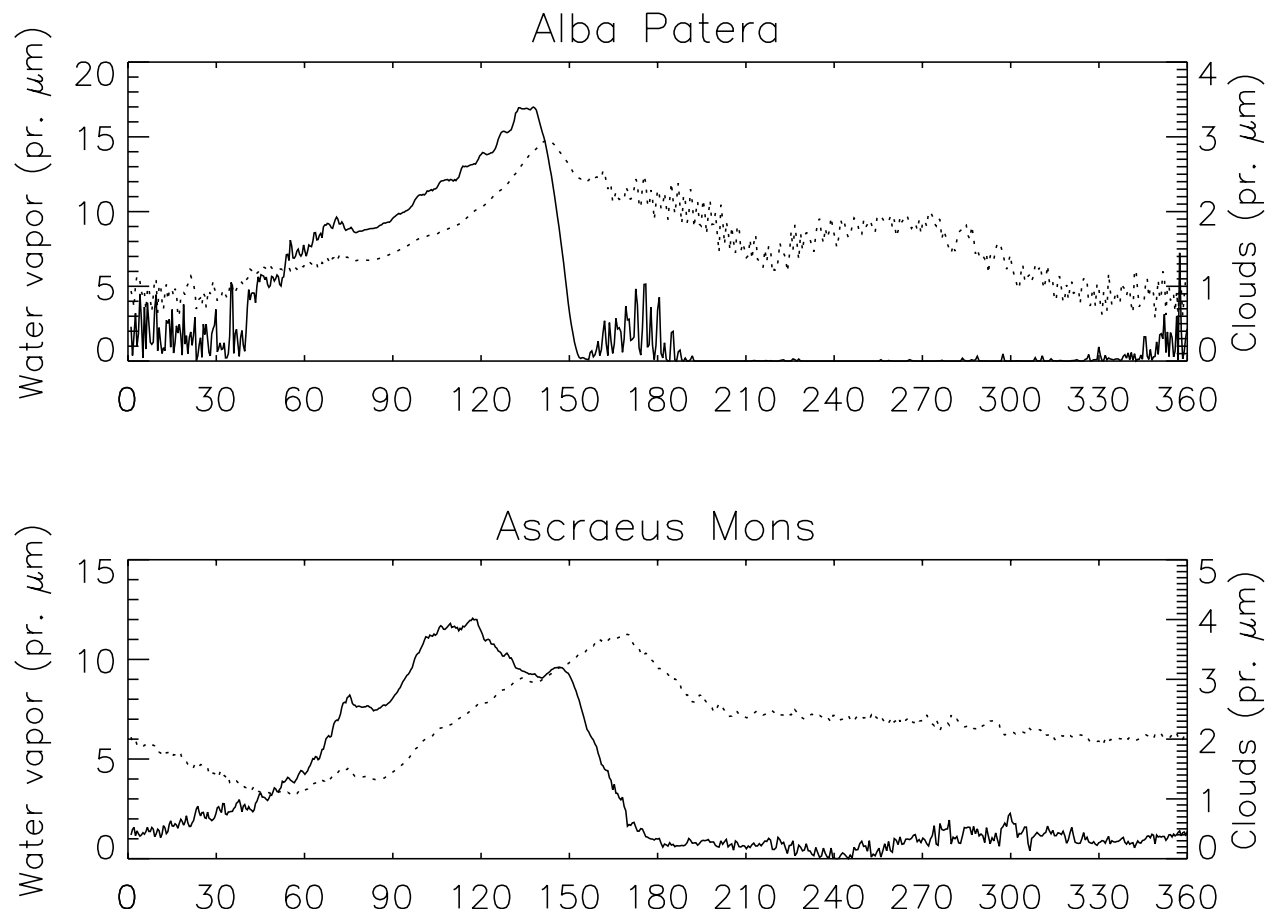

Figure 12. (top) Seasonal variation of the water vapor (dotted curve) and water ice cloud (bold curve) abundances at the location of the Alba Patera crater $\left(\sim 40^{\circ} \mathrm{N}, 110^{\circ} \mathrm{W}\right)$. Results given by our model. (bottom) Same as above but for Ascraeus Mons $\left(\sim 11^{\circ} \mathrm{N}, 105^{\circ} \mathrm{W}\right)$. 

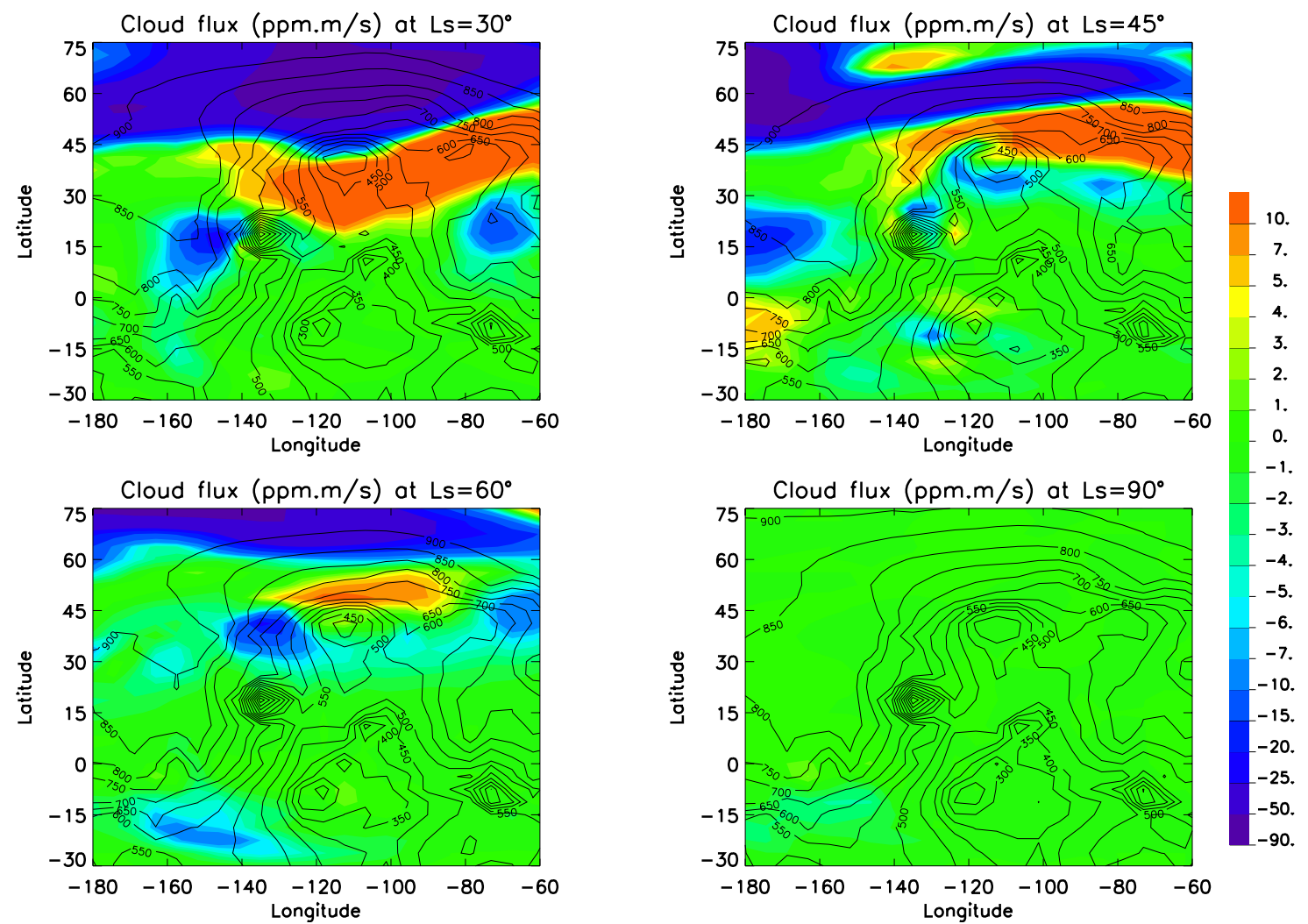

Figure 13. Meridional flux of water ice clouds generated by transient eddies at a pressure level of 3 mbar. Contours of iso-surface pressure have been superimposed to indicate topography.

presence of one spiral cloud, Wang and Ingersoll [2002] note that successive streaks usually follow within a two hour time interval. However, the structure shown by the model is clearly in keeping with Wang's description. Moreover, the GCM produces a similar seasonal behavior as the one observed, since these kind of clouds are mostly predicted near the end of northern fall.

[80] The role of these clouds in the water cycle should be substantial. While carrying water ice over great distances, streak clouds provide a means for the north polar cap to break with its isolation in the polar vortex. Through sedimentation, these clouds could supply a significant amount of water to the arctic region, and thus complement the quasi-solid return of water occurring later in spring.

[81] In contrast, streaks observed in the southern Polar Hood feature much thinner clouds, and their numbers are substantially lower than their northern counterparts [Wang and Ingersoll, 2002]. Although the GCM predicts that south Polar Hood clouds are thinner than in the north, we have not been able to detect any longitudinal structure in the results that resemble the spiral morphology prevailing in the north fall polar region.

\subsubsection{Polar Hood Passage Over the Viking Lander 2 Site}

[82] Viking Lander 2 meteorological data have revealed anomalous variations interpreted in terms of frontal systems associated with the advection of the Polar Hood over the VL2 landing site [Tillman et al., 1979]. These authors analyzed a 12-sol period of wind speed, temperature, pressure and opacity measurements to conclude on the likely passage of a cold front, carrying thick clouds with it. The discovery of such weather systems constitutes the first in-situ evidence of their presence in the Martian atmosphere. An isolated event of high opacity is obvious in the data, with a rapid obscuration of the surface surrounding the Lander, followed later by a more gradual opacity increase probably resulting from a global dust storm. However, the authors note that despite the prominence of this event, a series of fronts passing every 3 sols are also contained in the time series.

[83] In the absence of spectroscopic measurements, the hypothesis made by Tillman et al. [1979] of water ice clouds reducing surface illumination can only be speculative. However, we shall demonstrate below that this hypothesis is supported by our model. To do so, we have monitored the predictions made by the GCM at the location of VL2 for the same time frame analyzed by Tillman et al. [1979]. The results are shown in Figure 15. All the data presented in this graph have been filtered in frequency by applying a running average with a 1 day window. We did this to eliminate all the signals associated with diurnal variability, whether resulting from radiative adjustment or tides, and to focus on lower frequency signals such as those resulting from frontal systems passage (typically larger than 1 or 2 days).

[84] Tillman et al. [1979] list the criteria employed for the detection of cold fronts (which are the same as those used for Earth); winds shift from southerly to northerly, temperatures fall, and pressures rise. In Figure 15, we can see that this exact behavior is achieved by the model. The time 

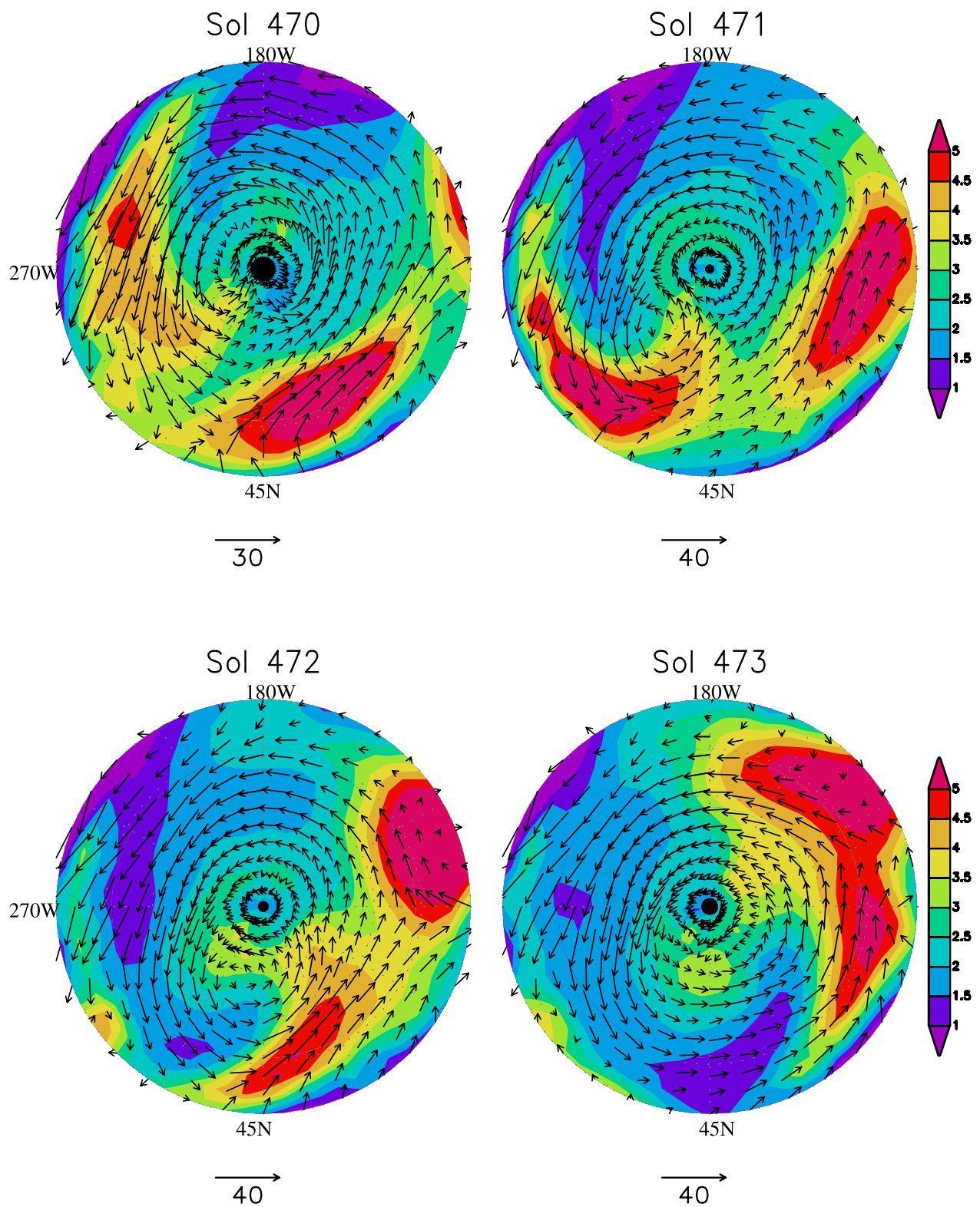

Figure 14. Stereographic projections of the North Polar region for four consecutive days (sols 470 to 473, corresponding to $\mathrm{L}_{s} \sim 241$ and 243, respectively). Shaded contours indicate water ice cloud abundances in pr. $\mu \mathrm{m}$. Arrows indicate wind directions and intensity (with a scaling, in $\mathrm{m} \mathrm{s}^{-1}$, indicated below each graph). The birth of a spiral in the western hemisphere (west longitudes are counted negative) is visible at a mean latitude of $60^{\circ}$ (top left panel). The spiral is then advected eastward while converging toward the pole.

frame monitored (15 days) allows for the passage of 4 successive cold fronts. A Fast Fourier Transformation of the GCM data (not shown here) shows that the power spectrum of either pressure, wind or temperature markedly peaks at a frequency of 0.3 cycle per day, and thus statistically validates the 3 day period that can be intuitively gleaned from Figure 15.

[85] We note that not only are the pressure, wind and temperature variations consistent with traveling frontal systems, but so too are variations in cloud opacity. Specifically, cloud opacity is strongly correlated with pressure, which indicates that the clouds are forming behind the cold front. The cloud abundances predicted by the model $(\sim 10$ pr. $\mu \mathrm{m})$ are consistent with what can been deduced from Viking Lander 2 images. The latter yield a visible opacity larger than 3 , which, when converted into water ice content $\left(M_{c} \sim\left(\tau r_{c} \rho_{i}\right) /\left(0.75 Q_{\text {ext }}\right)\right)$ gives 7-8 pr. $\mu \mathrm{m}$ (assuming particles of $4 \mu \mathrm{m}$ and an extinction efficiency coefficient $Q_{\text {ext }}$ of 2). This value can not be taken as representative of all clouds carried by cold fronts, since in this case, we are dealing with an isolated well-defined event, and consequently it may be more indicative of a maximum rather than an average behavior. Nonetheless, this phenomenon gives us some insight into how much water, in condensed form, can be potentially advected by such disturbances. 

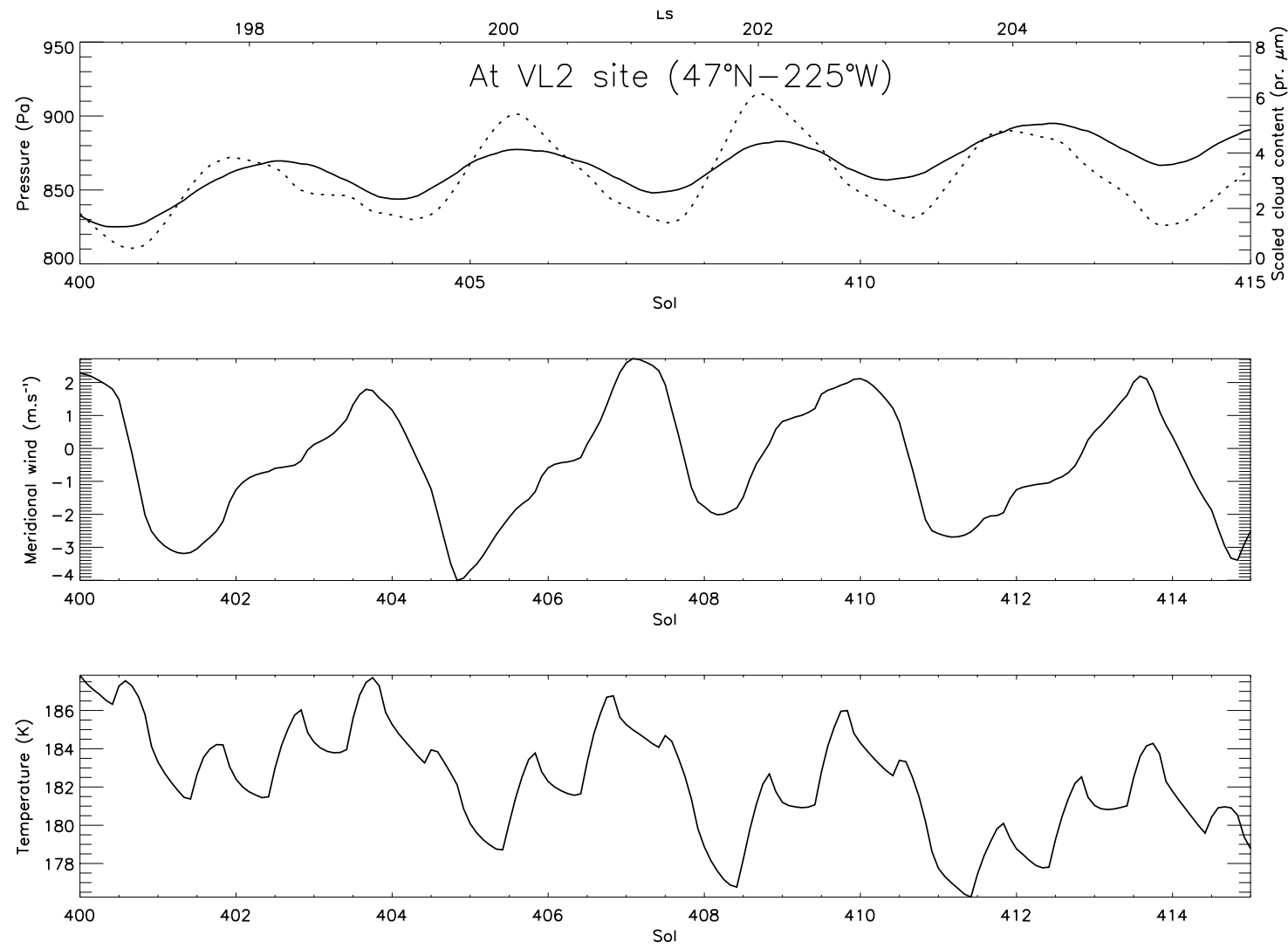

Figure 15. Time series of the pressure, cloud, wind, and temperature data produced by the model at the Viking Lander 2 site for the same time frame as that analyzed by Tillman et al. [1979]. In the top panel, pressure (bold line) is plotted with the cloud content scaled to a reference pressure of 6.1 mbar (dotted line). This scaling has been necessary to remove the effects of pressure variation on the integrated mass of clouds and thereby allow us to independently measure the true variation of cloud mass.

[86] However, some precaution must be taken when using the term "advected." This data series at a specific location does not provide conclusive evidence that these clouds, in reality or in the model, were actually a former component of the Polar Hood. Only a Lagrangian analysis could help us track their true origin. First, let's examine the potential distance that can be traveled by clouds. For a particle of a few microns lofted in the first 20 kilometers, it takes approximately $10^{6}$ seconds (on the order of ten days) to fall through an atmospheric scale height. Compared to the mean meridional velocity exhibited by Figure 15, which is roughly $3 \mathrm{~m} \cdot \mathrm{s}^{-1}$, clouds should be able to travel across 3,000 kilometers along a meridian (50 in a polar referential). This value appears to be more than required for Polar Hood clouds to be carried from high to midlatitude regions. Consequently, it is plausible that Polar Hood clouds could have been advected over the VL2 landing site.

[87] Still, this is not sufficient to confirm Tillman's hypothesis, since we can envision another possibility where such clouds could be formed locally, on the edge of the cold front, due to the effects of a cross-frontal circulation. In this case, wet and warm air masses south of the disturbance would be pushed upward, forcing water vapor to condense in the vicinity of the front where cold and warm air masses are merging together. Indeed, this type of mechanism has been invoked by Wang and Ingersoll [2002] to explain the shape of some spiral clouds that have been identified in many MOC images of the north polar region in summer. Nonetheless, this description does not fit with the data plotted in Figure 15. If we consider that the edge of the cold front corresponds to a positive pressure derivative, and thus a local minimum in the pressure series, cloud contents should be maximum at or near the time of this shift if they were forced by warm air uplifts. Figure 15 clearly shows that both pressure and clouds are almost exactly phased together, with clouds peaking right in the heart of the highpressure system.

[88] These considerations lead us to believe that frontal systems associated with baroclinic eddies can transport parts of the Polar Hood clouds southward to lower latitudes. To bring further support to this notion, Akabane et al. [1995] attribute the occurrence of bright spots moving eastward near the edge of the Polar Hood in late fall to the presence of cold fronts.

[89] Finally, such phenomena strongly suggest that atmospheric water ice can be transported over significant distances. Moreover, considering the cloud abundance inferred from VL2 data ( $7-8$ pr. $\mu \mathrm{m}$ to be compared to a globally averaged water content of roughly $10 \mathrm{pr} . \mu \mathrm{m}$ ) and that found in model results, this transport potentially involves a large reservoir of water. We argue below that it might actually be 


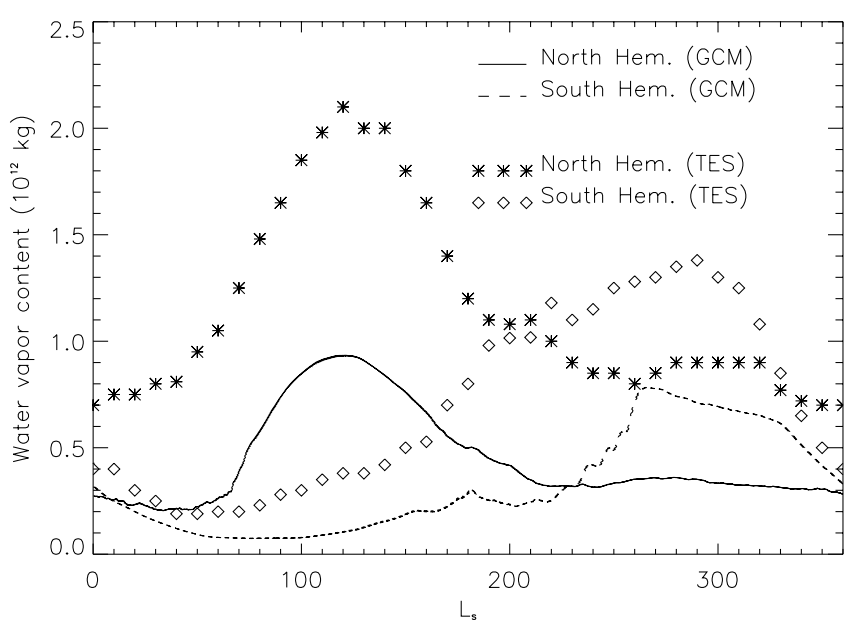

Figure 16. Comparison between the seasonal evolution of the integrated mass of water vapor in each hemisphere produced by the INSED case and that inferred from TES data.

one of the major components affecting the transport of water as a whole.

\section{How Do Clouds Affect the Water Cycle?}

[90] In order to assess the importance of the water ice cloud transport in the water cycle, it is necessary to understand how the distribution of water is affected by the cloud properties. In this regard, a critical issue concerns the ability of clouds to redistribute water vertically. To investigate this effect, we have performed a sensitivity study where cloud sedimentation properties have been set to their extremes of efficiency.

[91] The "Instantaneous Sedimentation" (INSED) experiment employs the same representation of clouds as that used by Haberle and Jakosky [1990]; i.e., any supersaturated excess of water vapor is transferred to the next lowest layer. In effect, this cloud scheme implies that condensation and sublimation are much faster than sedimentation, and that the cloud particles are so big that sedimentation itself is much faster than vertical mixing or advection. This approach excessively confines water vapor to the lowest layers.

[92] In the opposite extreme, the "No Sedimentation" (NOSED) experiment assumes that the cloud particles are so small that they are not subjected to gravitational settling. Of course, this type of simulation can not achieve a steady-state since the water holding capacity of the atmosphere becomes infinite. For this reason, we start the NOSED experiment from the last year of the Baseline simulation and run it for an additional year with cloud sedimentation switched off.

[93] As illustrated by Figure 16, the INSED experiment produces a much dryer water cycle, with global vapor amounts being lower by a factor of 2 than those observed and those produced by the Baseline simulation. The following sections discusses the main mechanisms responsible for the reduction in humidity subsequent to the assumption of instantaneous cloud precipitation.

\section{1. "Clancy Effect"}

[94] As a mobile reservoir for water, clouds not only change the global amount of humidity, they also change the way water is geographically distributed (Figure 17). In this figure, the north to south ratio of water vapor is plotted as a function of time, allowing us to explore the sensitivity of the cross-equatorial flow of water to changing cloud sedimentation properties.

[95] According to this graph, the effect of cloud sedimentation is most perceptible during northern spring and summer. This change in partitioning of water vapor between the northern and southern hemispheres is caused by the presence of the ECB in the lower levels of the Hadley cell upwelling zone (see Figure 18). Around aphelion, precipitation causes the water to be sequestered in the northern tropics where the ascending branch of the Hadley cell is located. This effect is illustrated in Figure 18. By transferring any supersaturated excess of water vapor to lower layers, the INSED experiment unrealistically enhances the vertical confinement of water vapor, whereas the Baseline simulation allows water ice clouds to be kept aloft at elevations corresponding to the southward moving branch of the Hadley cell. Here again, a comparison between the sedimentation timescale (approximately 1 week) and that of meridional advection (winds are predicted to be $\sim 3 \mathrm{~m} / \mathrm{s}$, which implies that roughly $2000 \mathrm{~km}$ can be covered over 1 week) shows that clouds can be entrained in the southward branch of the cell and cross the equator. Moreover, since the clouds are subjected to a strong diurnal cycle, the subsequent amount of water vapor subliming diurnally from ice crystals can be efficiently carried southward.

[96] The significant difference in predicted north to south humidity ratio between the different cloud schemes gives strong theoretical support to the influence of the aphelion cloud belt on the water flux between the north and south

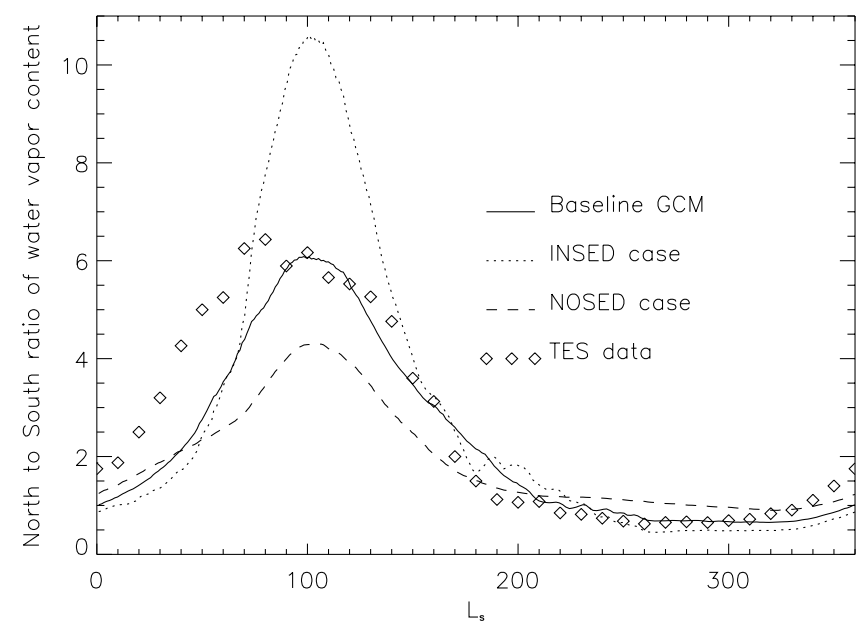

Figure 17. Seasonal evolution of the north to south ratio of water vapor as given by different versions of the model, to be compared with that deduced from TES observations. The differences between the models around aphelion is mostly due to a modulation of the water transport by the overturning circulation between the two hemispheres. Hence it can be seen that potentially, the transport of clouds near aphelion can decrease the north to south asymmetry of humidity from a value exceeding 10 to a value of 4. Consequently, the cross-equatorial flow of water at that season is mainly controlled by cloud sedimentation in the northern tropics. 

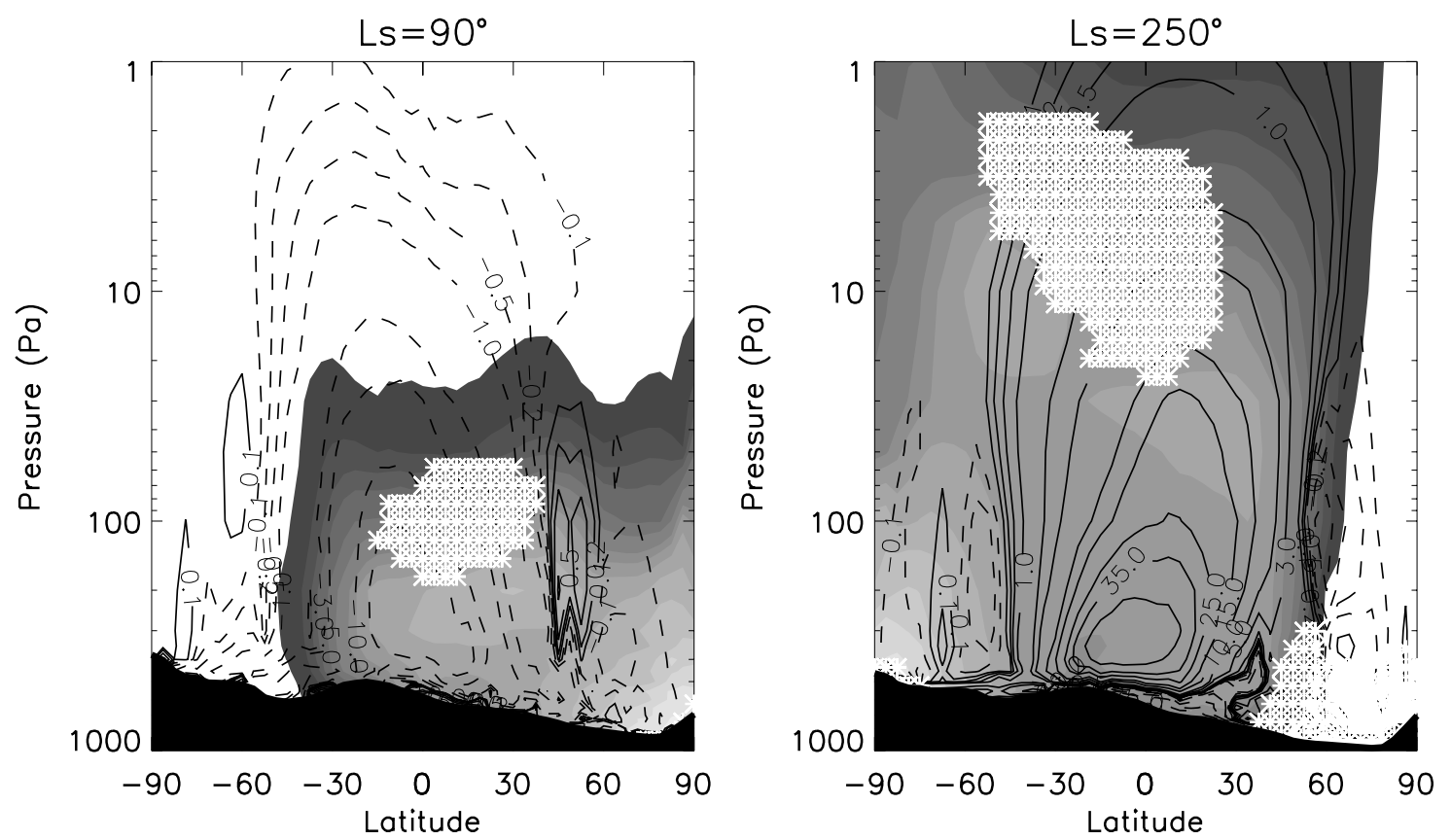

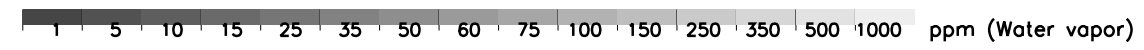

Figure 18. Latitudinal cross sections of water vapor (shaded) and mass stream functions (contours in $10^{8} \mathrm{~kg} / \mathrm{s}$ ) at two seasons. Water ice cloud locations inferred from the model are indicated by the white stars.

hemispheres [Clancy et al., 1996]. Figure 17 allows us to quantify the so-called "Clancy effect." It appears however that the actual sequestration of water in the northern tropics generated by the clouds is closer in effect to the NOSED situation than the INSED situation, as the INSED curve exhibits a larger gap with the Baseline curve than the NOSED does. These results mean that clouds rather favor water transfer to the south hemisphere than the opposite, given the potential they have to retain moisture in the summer north hemisphere.

[97] If cloud particles were to exhibit negligible sizes (NOSED), the north to south ratio during northern summer would be around 4 . The NOSED case could also reflect a dusty, though unlikely, aphelion season, where the ECB would form at much higher altitudes (Figure 18) like during perihelion season where the differences among the three models become almost negligible. If cloud particles were large enough to precipitate very quickly, the north to south ratio would increase up to 11 , whereas the actual ratio, well fitted by our baseline case, exhibits values around 6 . From these values, we can therefore conclude that the mass of water contained in clouds participates just as much as water vapor itself in the southward migration of moisture during northern summer. Nonetheless, cloud sedimentation is also responsible for retaining the equivalent of $50 \%$ of the southward flow of water in the northern tropics.

[98] Figure 17 also demonstrates that the north to south ratio is not well reproduced between $\mathrm{Ls}=330^{\circ}$ and $60^{\circ}$, regardless of the assumptions made for cloud sedimentation. As mentioned previously, this is due to our predictions of an overly wet southern hemisphere during the same period.
Part of the reason involves the phasing in the recession of the southern seasonal cap of $\mathrm{CO}_{2}$. The GCM predicts a faster recession than observed. Consequently, water vapor is released too soon and becomes available for extrapolar transport over a longer timescale. In addition, the consequences of neglecting regolith adsorption might be of some importance, since this process should potentially help in reducing the amount of atmospheric water vapor during this season [Böttger, 2003].

\subsection{Polar Hood Effect}

[99] Figure 19 provides a more detailed assessment of the change in geographic distribution of water due to cloud transport. It can be seen that clouds (as opposed to the INSED case where clouds can not trace circulation) mostly amplify water vapor abundances in the low to mid latitude regions. The northern hemisphere benefits even more from this effect, which implies that the increase in the southward flow of water during northern summer is not the main consequence of allowing cloud advection. As expected, the Baseline case results are in very good agreement with TES observations.

[100] Figure 20 displays a contour map of the ratio of the water vapor abundances predicted by the Baseline case onto that produced by the INSED case. With this figure, we are able to identify when and where the transport of clouds has the most significant impact. On an annual basis, there are five different zones associated to higher ratio values and thus to a cloud-induced moistening of the atmosphere. In fact, we can separate these five events into two subcategories, each of which are related to a particular mechanism. 


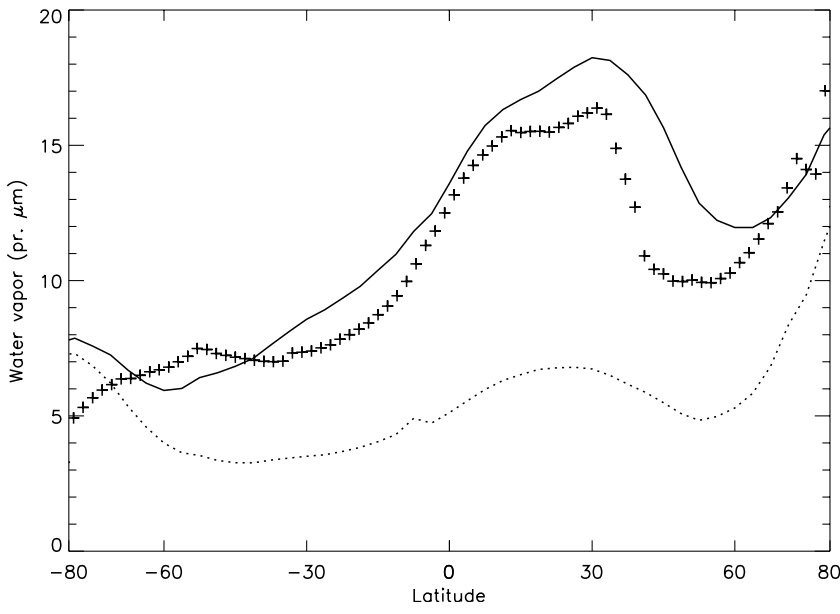

Figure 19. Annual averages of the zonal mean water vapor abundances. Bold line: Baseline GCM version. Dotted line: INSED case. Crosses: TES observations.

The first category includes the black portion of Figure 20 located in the low to midlatitudes of the southern hemisphere between $\mathrm{Ls}=30^{\circ}$ and $\mathrm{Ls}=150^{\circ}$. It also comprises the black portion of the north polar region between $\mathrm{Ls}=$ $240^{\circ}$ and $\mathrm{Ls}=330^{\circ}$.

[101] This first category is related to cloud transport via the solsticial Hadley cells, which has been discussed in the previous section. The latitudinal offset between these two zones of high ratios reflects the difference between the aphelion and the perihelion season. As the latter implies cloud formation at high altitudes in the upwelling zone of the southern summer tropics, the region where clouds partially sublime in the downwelling branch of the winter hemisphere is located more poleward than during aphelion season.

[102] The second category includes the three remaining high ratio portions, i.e., that prevailing in the $30^{\circ} \mathrm{N}$ to $70^{\circ} \mathrm{N}$ between $\mathrm{Ls}=0^{\circ}$ and $\mathrm{Ls}=90^{\circ}$, that occurring between Ls $=$ $150^{\circ}$ and $\mathrm{Ls}=240^{\circ}$ in the vicinity of the receding south cap, and that located between $\mathrm{Ls}=150^{\circ}$ and $\mathrm{Ls}=210^{\circ}$ in the $\left[30^{\circ} \mathrm{N}, 60^{\circ} \mathrm{N}\right]$ region. This category involves water ice cloud advection by the residual mean circulation, namely atmospheric waves. During seasonal cap recessions, the descent of cold fronts into the equatorward regions entrains a large amount of water ice clouds formed in the polar latitudes. This balances the flow of water vapor in the opposite direction, which follows warm front trajectories. In the INSED simulation, water vapor is instantaneously trapped over the edge of the seasonal cap due to a rapid reduction of the water holding capacity of poleward flowing air masses, precluding any returning flow of water to lower latitudes. In the Baseline simulation, however, polar cloud particles remain aloft long enough to be advected equatorward, and are therefore able to release their water vapor content in the warmer tropical regions. This mechanism works in both hemispheres and explains the presence of the high ratio portions along the receding caps. This process not only works during polar cap recession, but also during their formation, though less efficiently, the black portion located along the expanding north cap around equinox is evidence of this.

[103] It is worth mentioning that an apparent hemispheric asymmetry produces the more efficient build up of water vapor in the northern hemisphere (Figure 19). As discussed in the previous sections, it is caused by a more intense

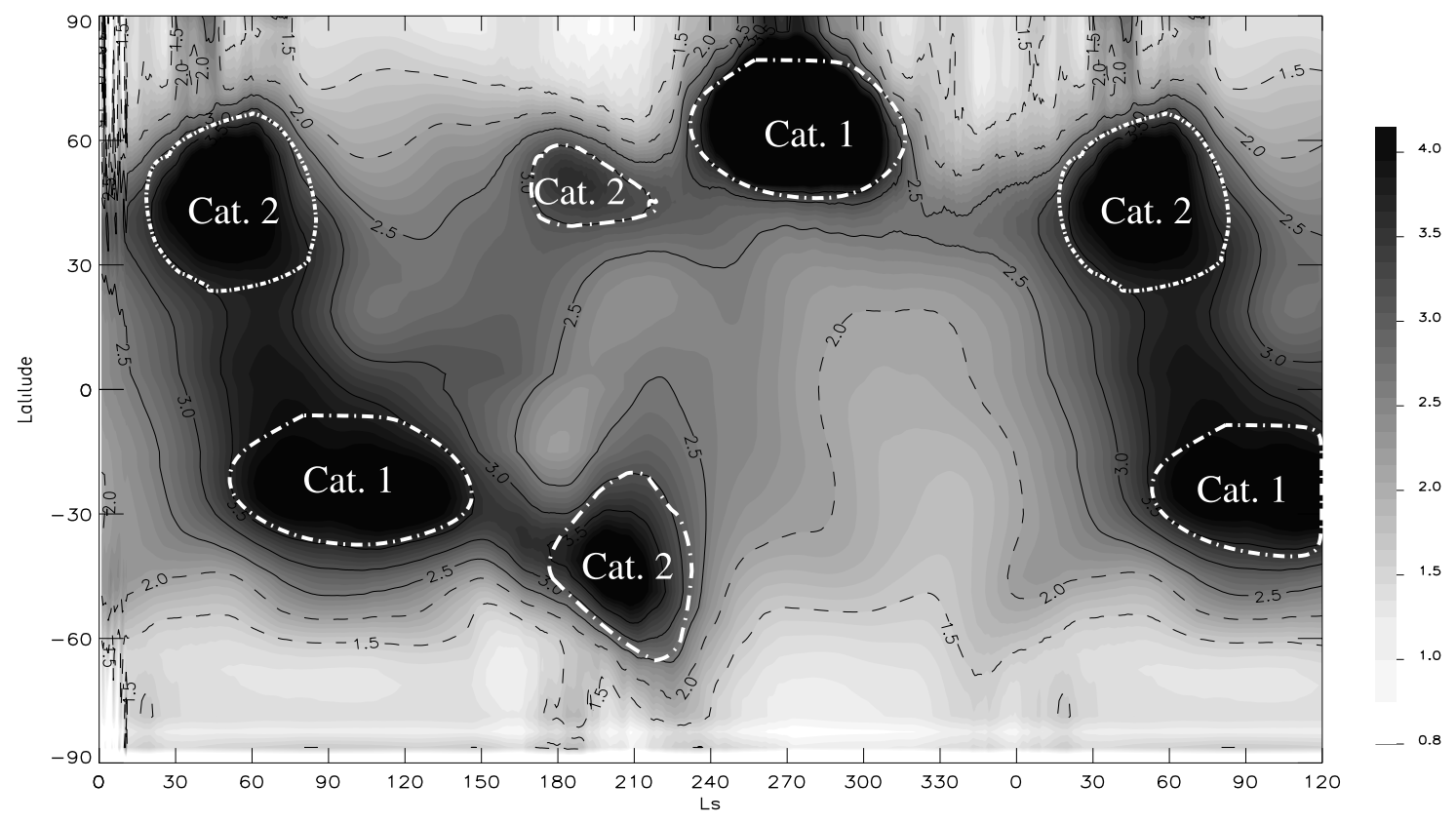

Figure 20. Ratio of the water vapor abundances predicted by the Baseline version of the GCM onto that predicted by the INSED case. Darker areas indicate increasing ratios and thus indicate where the transport of clouds is associated with an enrichment of water vapor. The globally and annually averaged value of the ratio is 2.5 . The two different categories of high ratio values discussed in the text have been labeled here for clarity. 
horizontal mixing in the north than in the south, where the baroclinic wave activity is predicted to be weaker.

[104] In summary, the equatorward transport of Polar Hood clouds during the recession and, to a lesser extent, during the expansion of the polar caps, acts at the expense of the water budgets of the polar regions. By counterbalancing the poleward flow of water vapor, cloud advection reduces the net transport to the poles. In some aspects, this mechanism can be considered as a way to delay the return of water to the north permanent cap. Once combined with the cross-equatorial migration of clouds around aphelion, cloud transport (and not cloud themselves) behaves as a moistening agent of the Martian atmosphere which explains the global reduction of humidity exhibited by the INSED simulation.

\section{Conclusion}

[105] In this paper, we have analyzed the origin of the main cloud formations in the Martian atmosphere by means of a MGCM water cycle model. In contrast with the study of Richardson et al. [2002], we have found it possible to reproduce the observed Martian hydrological cycle with reasonable assumptions for the cloud microphysical properties. Our approach of predicting cloud particle size clearly provides a significant improvement over simplified cloud schemes where particle radius are held constant. To bring an evidence to this statement, we have conducted an additional water cycle simulation where cloud sedimentation was computed for a constant $6 \mu \mathrm{m}$ cloud particle radius. The results of this experiment (not shown) indicate an important degradation of the quality of the model predictions, with a $50 \%$ increase in the overall humidity of the atmosphere, implying a water vapor content which by far exceeds TES observations and thus the results of our Baseline run. This value of $6 \mu \mathrm{m}$ was chosen by taking the mean cloud particle radius of the Equatorial Cloud Belt $(3-4 \mu \mathrm{m})$ and by applying the scaling factor of 1.5 discussed previously. These simulation results are in line with Richardson et al. [2002] who obtained an overly wet Martian atmosphere with a constant $2 \mu \mathrm{m}$ particle size, an issue that Richardson et al.'s corrected by using an unrealistic $15 \mu \mathrm{m}$ particle radius. This tends to show that assuming the microphysical properties of the ECB to be representative of the whole cloud cycle is misleading. Letting the model predict particle size leads to the presence of large icy crystals in the clouds of the Polar Hoods. As shown previously, the importance of these clouds are probably as large as is the ECB in modulating the seasonal behavior of the water cycle. While it is tempting to conclude that Richardson's model could obtain results similar to ours if using our cloud scheme, it is fair to mention that other factors pertaining to different aspects of the model might be also involved. Among all possible differences, Richardson et al. [2002] use a different ground ice sublimation scheme; their MGCM is forced by an interactive dust cycle (compared to our prescribed dust input).

[106] However, our validated water cycle model will allow further study of water-related processes, like the first three-dimensional photochemical study of the Martian atmosphere recently conducted by Lefèvre et al. [2004].

[107] We have shown that the control exerted by dynamical phenomena on the cloud distribution differs between the tropical belt region and the middle to high latitude regions. In the tropics, the cycle of cloudiness reflects the annual cycle of insolation (combined with changes in dust loading) and the seasonal variations of the mean meridional circulation. The Equatorial Cloud Belt appears weakly sensitive to the level of humidity, but is rather driven by the large scale upward motion of the northern tropics and a reduced atmospheric heating.

[108] The seasonal cycle of the Polar Hoods exhibits comparable patterns in both hemispheres, with markedly thicker clouds forming during the onset and the decay of the polar vortices. It appears that atmospheric wave activity controls in large part the behavior of the Polar Hoods. Eddy activity increases near the end of summer and reaches a first maximum at equinox. The associated horizontal mixing supplies significant amounts of water vapor that are extracted from the low latitude regions and promote cloud formation in the polar regions. The following fall and winter seasons see a reduction in wave activity, which added to the possible effects of polar warming events, are responsible for the minimum in Polar Hood opacity around solstice. Then, as spring approaches, eddy activity resumes and reintensifies cloud formation such that the thickest clouds of the Polar Hood cycle are obtained during cap recession.

[109] We have assessed the role of cloud transport and its effects on the seasonal and latitudinal distribution of water vapor. To do so, we have supplemented our Baseline simulation with other experiments where cloud sedimentation properties have been altered. In this way, we have been able to demonstrate the critical role played by clouds in the transport of water as a whole. Despite the low fraction of clouds in the atmospheric reservoir of water, they occur at key locations and key seasons of the water cycle, where they can regulate the cross-equatorial flow of water as well as the exchange of water between the tropics and the polar regions. The cloud sedimentation timescale is long enough to allow ice particles to be advected by meridional winds, an effect which generally acts against the return of water to the north permanent cap and forces an equilibrium state of the atmosphere to be globally much wetter.

[110] Finally, we have been able to reproduce the water cycle without including an adsorbing regolith. This would seem to indicate a minor role for the regolith in the present water cycle. However, this is the exact opposite conclusion reached by Böttger [2003] who also used a general circulation model to study the Martian water cycle, and who could not reproduce the observed water cycle without an adsorbing regolith. Our feeling is that neither model is mature enough yet to fully assess the role of the regolith. Böttger did not predict cloud particle size, and in his model water was allowed to exchange between the regolith and atmosphere even when covered with $\mathrm{CO} 2$ ice. And there is always the vexing problem of not knowing the actual adsorptive properties of the regolith itself. In our model, the cloud scheme does not include two-way dust/ice interactions or the radiative effects of clouds. And our South $\mathrm{CO} 2$ cap retreats too quickly which leads to a wetter southern spring than is observed. Perhaps this may be a place where the regolith can play a role. But what is clear from Böttger's work, our work, and that of Richardson and Wilson [2002], is that clouds play a major role in the seasonal and spatial distribution of water. Therefore, until 
we have confidence in our ability to predict them, the role of the regolith cannot be ascertained.

[111] Acknowledgments. The authors are indebted to J. R. Hollingsworth for sharing its expertise on the atmospheric transport phenomena, as well as to Mike Smith, Jennifer Benson and Todd Clancy for having kindly shared their data sets. We also wish to thank John Wilson and an anonymous reviewer for their constructive comments on the paper. This paper was written while F. M. was holding a National Research Council award to conduct research at the NASA Ames Research Center. In addition, a significant part of this work has been also supported by a grant from the Programme National de Planétologie (France).

\section{References}

Akabane, T., Y. Saito, K. Iwasaki, and L. J. Martin (1995), The north polar hood in early autumn on Mars, Astron. Astrophys., 304, 595-601.

Bass, D. S., and D. A. Paige (2000), Variability of Mars' north polar water ice cap. II. Analysis of Viking IRTM and MAWD, Icarus, 144, 397-409.

Bass, D. S., K. Herkenhoff, and D. A. Paige (2000), Variability of Mars' north polar water ice cap. I. Analysis of Mariner 9 and Viking Orbiter imaging data, Icarus, 144, 382-396.

Benson, J. L., B. P. Bonev, P. B. James, K. J. Shan, B. A. Cantor, and M. A. Caplinger (2003), The seasonal behavior of water ice clouds in the Tharsis and Valles Marineris regions of Mars: Mars Orbiter Camera observations, Icarus, 165, 34-52.

Böttger, H. M. (2003), Modelling the water cycle on mars, Ph.D. thesis, University of Oxford, Oxford, England.

Clancy, R. T., A. W. Grossman, M. J. Wolff, P. B. James, D. J. Rudy, Y. N. Billawala, B. J. Sandor, S. W. Lee, and D. O. Muhleman (1996), Water vapor saturation at low latitudes around aphelion: A key to Mars climate?, Icarus, 122, 36-62.

Clancy, R. T., M. J. Wolff, and P. R. Christensen (2003), Mars aerosol studies with the MGS TES emission phase function observations: Optical depths, particle sizes, and ice cloud types versus latitude and solar longitude, J. Geophys. Res., 108(E9), 5098, doi:10.1029/2003JE002058

Colaprete, A., and O. B. Toon (2000), The radiative effects of Martian water ice clouds on the local atmospheric temperature profile, Icarus, $145,524-532$

Colaprete, A., O. B. Toon, and J. A. Magalhães (1999), Cloud formation under Mars Pathfinder conditions, J. Geophys. Res., 104, 9043-9053.

Conrath, B. J. (1975), Thermal structure of the Martian atmosphere during the dissipation of dust storm 1971, Icarus, 24, 34-46.

Flasar, F. M., and R. M. Goody (1976), Diurnal behaviour of water on Mars, Planet. Space Sci., 24, 161-181.

Forget, F., F. Hourdin, R. Fournier, C. Hourdin, O. Talagrand, M. Collins, S. R. Lewis, P. L. Read, and J. Huot (1999), Improved general circulation models of the Martian atmosphere from the surface to above $80 \mathrm{~km}$, J. Geophys. Res., 104, 24,155-24,176.

Forget, F., Y. Wanherdrick, and S. R. Lewis (2001), Validation of the Mars General Circulation Model and Climate Database with new spacecraft observations, in Work Package 7, Tech. Note 11369/95/NL/JG, Eur. Space Agency, Paris. (Available at http://www-mars.lmd.jussieu.fr/mars/esadoc/ lmd/esadoc.html.)

Haberle, R. M., and B. M. Jakosky (1990), Sublimation and transport of water from the north residual polar cap on Mars, J. Geophys. Res., 95, $1423-1437$

Haberle, R., M. Joshi, J. Murphy, J. Barnes, J. Schofield, G. Wilson, M. Lopez-Valverde, J. Hollingsworth, A. Bridger, and J. Schaeffer (1999), General circulation model simulations of the Mars Pathfinder atmospheric structure investigation/meteorology data, J. Geophys. Res., 104(E4), 8957-8974.

Hinson, D. P., and R. J. Wilson (2004), Temperature inversions, thermal tides, and water ice clouds in the Martian tropics, J. Geophys. Res., 109, E01002, doi:10.1029/2003JE002129.

Houben, H., R. M. Haberle, R. E. Young, and A. P. Zent (1997), Modeling the Martian seasonal water cycle, J. Geophys. Res., 102, 9069-9083.

Hourdin, F., and A. Armengaud (1999), On the use of finite volume methods for atmospheric advection of trace species: I. Test of various formulations in a General Circulation Model, Mon. Weather Rev., 127, 822837.

Jacobson, M. Z. (1997), Numerical techniques to solve condensational and dissolutional growth equations when growth is coupled to reversible reactions, Aerosol Sci. Technol., 27, 491-498.

Jakosky, B. M. (1983), The role of seasonal reservoir in Mars water cycle: II. Coupled model of the regolith, the polar caps and atmospheric transport, Icarus, 55, 19-39.

Jakosky, B. M., and C. B. Farmer (1982), The seasonal and global behavior of water vapor in the Mars atmosphere: Complete global results of the
Viking atmospheric water detector experiment, J. Geophys. Res., 87, $2999-3019$.

James, P. B. (1985), The Martian hydrologic cycle-Effects of $\mathrm{CO}_{2}$ mass flux on global water distribution, Icarus, 64, 249-264.

James, P. B. (1990), The role of water ice clouds in the Martian hydrologic cycle, J. Geophys. Res., 95, 1439-1445.

James, P. B., J. F. Bell, R. T. Clancy, S. W. Lee, L. J. Martin, and M. J. Wolff (1996), Global imaging of Mars by Hubble Space Telescope during the 1995 opposition, J. Geophys. Res., 101, 18,883-18,890.

Kahn, R. (1984), The spatial and seasonal distribution of Martian clouds and some meteorological implications, J. Geophys. Res., 89, 6671-6688. Kahn, R. (1990), Ice haze, snow, and the Mars water cycle, J. Geophys. Res., 95, 14,677-14,693.

Lefèvre, F., S. Lebonnois, F. Montmessin, and F. Forget (2004), Threedimensional modeling of ozone on Mars, J. Geophys. Res., 109, E07004, doi:10.1029/2004JE002268.

Liu, J., M. I. Richardson, and R. J. Wilson (2003), An assessment of the global, seasonal, and interannual spacecraft record of Martian climate in the thermal infrared, J. Geophys. Res., 108(E8), 5089, doi:10.1029/ 2002JE001921.

Mellon, M. T., B. M. Jakosky, H. H. Kieffer, and P. R. Christensen (2000), High-resolution thermal inertia mapping from the Mars Global Surveyor Thermal Emission Spectrometer, Icarus, 148, 437-455.

Michelangeli, D. V., O. B. Toon, R. M. Haberle, and J. B. Pollack (1993), Numerical simulations of the formation and evolution of water ice clouds in the Martian atmosphere, Icarus, 100, 261-285.

Montmessin, F., P. Rannou, and M. Cabane (2002), New insights into Martian dust distribution and water-ice cloud microphysics, J. Geophys. Res., 107(E6), 5037, doi:10.1029/2001JE001520.

Neumann, G. A., D. E. Smith, and M. T. Zuber (2003), Two Mars years of clouds detected by the Mars Orbiter Laser Altimeter, J. Geophys. Res., 108(E4), 5023, doi:10.1029/2002JE001849.

Paige, D. A., and K. D. Keegan (1994), Thermal and albedo mapping of the polar regions of Mars using Viking thermal mapper observations: 2. South polar region, J. Geophys. Res., 99(E12), 25,993-26,013.

Paige, D. A., J. E. Bachman, and K. D. Keegan (1994), Thermal and albedo mapping of the polar regions of Mars using Viking thermal mapper observations: 1. North polar region, J. Geophys. Res., 99(E12), $25,959-25,991$.

Palluconi, F. D., and H. H. Kieffer (1981), Thermal inertia mapping of Mars from $60 \mathrm{deg} \mathrm{S}$ to $60 \mathrm{deg} \mathrm{N}$, Icarus, 45, 415-426.

Peixoto, J. P., and A. H. Oort (1992), Physics of Climate, Am. Inst. of Phys., New York.

Pleskot, L. K., and D. E. Miner (1981), Time variability of Martian bolometric albedo, Icarus, 45, 179-201.

Pollack, J. B., R. M. Haberle, J. Schaeffer, and H. Lee (1990), Simulations of the general circulation of the Martian atmosphere: 1. Polar processes, J. Geophys. Res., 95, 1447-1473.

Richardson, M. I., and R. J. Wilson (2002), Investigation of the nature and stability of the Martian seasonal water cycle with a general circulation model, J. Geophys. Res., 107(E5), 5031, doi:10.1029/2001JE001536.

Richardson, M. I., R. J. Wilson, and A. V. Rodin (2002), Water ice clouds in the Martian atmosphere: General circulation model experiments with a simple cloud scheme, J. Geophys. Res., 107(E9), 5064, doi:10.1029/ 2001JE001804.

Rodin, A. V. (2002), On the moment method for the modeling of cloud microphysics in rarefied turbulent atmospheres: I. Condensation and mixing, Sol. Syst. Res., 36, 97-106.

Rodin, A. V., R. T. Clancy, and R. J. Wilson (1999), Dynamical properties of Mars water ice clouds and their interactions with atmospheric dust and radiation, Adv. Space Res., 23, 1577-1585.

Rossow, W. B. (1978), Cloud microphysics: Analysis of the clouds of Earth, Venus, Mars, and Jupiter, Icarus, 36, 1-50.

Sadourny, R., and K. Laval (1984), January and July performance of the LMD general circulation model, in New Perspectives in Climate Modeling, pp. $173-197$, edited by A. Berger and C. Nicolis, Elsevier Sci., New York.

Savijärvi, H. (1991), Radiative fluxes on a dustfree Mars, Contrib. Atmos. Phys., 64, 103-112.

Savijärvi, H. (1995), Mars boundary layer modeling: Diurnal moisture cycle and soil properties at the Viking Lander 1 site, Icarus, 117, 120-127.

Schulz, M., Y. J. Balkanski, W. Guelle, and F. Dulac (1998), Role of aerosol size distribution and source location in a three-dimensional simulation of a Saharan dust episode tested against satellite-derived optical thickness, J. Geophys. Res., 103, 10,579-10,592.

Smith, D. E., et al. (1999), The global topography of Mars and implications for surface evolution, Science, 284, 1495-1503.

Smith, M. D. (2002), The annual cycle of water vapor on Mars as observed by the Thermal Emission Spectrometer, J. Geophys. Res., 107(E11), 5115, doi:10.1029/2001JE001522. 
Smith, M. D. (2004), Annual variability in TES atmospheric observations of Mars during 1999-2003, Icarus, 167, 148-165.

Smith, M. D., J. C. Pearl, B. J. Conrath, and P. R. Christensen (2001), One Martian year of atmospheric observations by the Thermal Emission Spectrometer, Geophys. Res. Let., 28, 4263-4266.

Sprague, A. L., D. M. Hunten, R. E. Hill, L. R. Doose, and B. Rizk (2001), Water vapor abundances over Mars north high latitude regions: 19961999, Icarus, 154, 183-189.

Tamppari, L. K., R. W. Zurek, and D. A. Paige (2000), Viking era water-ice clouds, J. Geophys. Res., 105, 4087-4107.

Tamppari, L. K., R. W. Zurek, and D. A. Paige (2003), Viking-era diurnal water-ice clouds, J. Geophys. Res., 108(E7), 5073, doi:10.1029/ 2002JE001911.

Tillman, J. E., R. M. Henry, and S. L. Hess (1979), Frontal systems during passage of the Martian north polar hood over the Viking Lander 2 site prior to the first 1977 dust storm, J. Geophys. Res., 84, 2947-2955.

Titus, T. N., and H. H. Kieffer (2003), Temporal and spatial distribution of $\mathrm{CO}_{2}$ snow and ice (abstract), paper presented at Sixth International Conference on Mars, Lunar and Planet. Inst., Pasadena, Calif.

Vasavada, A. R., J. Williams, D. A. Paige, K. E. Herkenhoff, N. T. Bridges, R. Greeley, B. C. Murray, D. S. Bass, and K. S. McBride (2000), Surface properties of Mars' polar layered deposits and polar landing sites, J. Geophys. Res., 105, 6961-6970.

Wang, H., and A. P. Ingersoll (2002), Martian clouds observed by Mars Global Surveyor Mars Orbiter Camera, J. Geophys. Res., 107(E10), 5078, doi:10.1029/2001JE001815.
Wolff, M. J., J. F. Bell, P. B. James, R. T. Clancy, and S. W. Lee (1999), Hubble Space Telescope observations of the Martian aphelion cloud belt prior to the Pathfinder mission: Seasonal and interannual variations, J. Geophys. Res., 104, 9027-9042.

Zent, A. P., R. M. Haberle, H. C. Houben, and B. M. Jakosky (1993), A coupled subsurface-boundary layer model of water on Mars, J. Geophys. Res, 98, 3319-3337.

Zurek, R. W., J. R. Barnes, R. M. Haberle, J. B. Pollack, J. E. Tillman, and C. B. Leovy (1992), Dynamics of the atmosphere of Mars, in Mars, edited by H. H. Kieffer et al., chap. 26, pp. 835-933, Univ. of Ariz. Press, Tucson.

M. Cabane, Service d'Aéronomie, Institut Pierre Simon Laplace, Université Pierre et Marie Curie, Tour 45, Couloir 45-46, 4ème Etage (Boîte 102), 4 Place Jussieu, 75252 Paris Cédex 05, France.

F. Forget, Laboratoire de Météorologie Dynamique, Institut Pierre Simon Laplace, Université Pierre et Marie Curie, Tour 45, 3ème Etage, BP99, 4 Place Jussieu, 75252 Paris Cédex 05, France.

R. M. Haberle and F. Montmessin, Space Science Division, NASA Ames Research Center, MS 245-3, Moffett Field, CA 94035-1000, USA. (fmontmessin@mail.arc.nasa.gov)

P. Rannou, Service d'Aéronomie, Institut Pierre Simon Laplace, Rduit de Verrires, BP 3, Route des Gatines, 91371 Verrières le Buisson Cédex, France. 